\title{
SÍNTESE DE $\beta$-D-GALACTOPIRANOSÍDEOS DE ARILA DIMÉRICOS PARA AVALIAÇÃO DE SUA INTERAÇÃO COM A LECTINA DE Erythrina cristagalli
}

\author{
Rute Cunha Figueiredo, Nádia Burkowski Meyer, Maria Auxiliadôra Fontes Prado e Ricardo José Alves* \\ Departamento de Produtos Farmacêuticos, Faculdade de Farmácia, Universidade Federal de Minas Gerais, Av. Antônio Carlos, \\ 6627, 31270-901 Belo Horizonte - MG, Brasil \\ Javier Rojo \\ Grupo de Carbohidratos, Instituto de Investigaciones Químicas, CSIC, Américo Vespucio 49, E-41092 Sevilla, Espanha
}

Recebido em 31/12/08; aceito em 13/4/09; publicado na web em 22/9/09

\begin{abstract}
SYNTHESIS OF DIMERIC ARYL $\beta$-D-GALACTOPYRANOSIDES FOR THE EVALUATION OF THEIR INTERACTION
WITH THE Erythrina cristagalli LECTIN. The synthesis of two new D-galactose-based dimers having a 1,4-butanediamine spacer is reported aiming at the evaluation of their interaction with the Erythrina cristagalli lectin. The title compounds were prepared in four and five steps from 2,3,4,6-tetra- $O$-acetyl- $\beta$-D-galactopyranoside bromide, in $20 \%$ and $15 \%$ overall yield, respectively, using the Doebner modification of the Koenavenagel reaction as the key sep. The lectin-carbohydrate interaction could be evaluated for only one dimer, due to solubility problems. A twofold enhancement of affinity was observed, compared to the corresponding monovalent ligand.
\end{abstract}

Keywords: D-galactopyranose; glycodendrimers; Erythrina cristagalli.

\section{INTRODUÇÃO}

Os glicoconjugados como as glicoproteínas e os glicolipídios possuem um papel importante em vários processos biológicos relacionados com reconhecimento e adesão celular, como inflamação, infecção e mestástase. ${ }^{1,2}$ As forças que regem esses processos de reconhecimento e adesão são interações complexas entre as porções carboidrato de um glicoconjugado e as lectinas, proteínas que reconhecem especificamente carboidratos.

Usualmente, a afinidade das lectinas por monossacarídeos é fraca. Entretanto, na natureza, essas interações são multivalentes. A multivalência está associada ao reconhecimento de múltiplas cópias de carboidrato apresentadas a múltiplas cópias de lectinas presentes na superfície celular. ${ }^{3}$ A multivalência aumenta a força e a seletividade das interações carboidrato-lectinas. ${ }^{4}$

Substâncias multivalentes sintéticas derivadas de carboidratos, denominadas genericamente glicodendrímeros, ${ }^{4}$ mimetizam a interação dos glicoconjugados com lectinas no sistema biológico. Há, portanto, grande interesse na obtenção dessas substâncias, para avaliação de suas interações com lectinas. ${ }^{1,4-8}$ Tem-se demonstrado que a eficiência das interações entre glicodendrímeros e lectinas depende de vários fatores, como o número de unidades de carboidratos presentes na molécula (multivalência), a distância entre essas unidades e a flexibilidade do dendrímero. ${ }^{9-11}$ De modo geral, aumentando-se a multivalência aumenta-se a eficiência da interação carboidratolectina. ${ }^{12-14}$ Entretanto, isso nem sempre é o caso. Há diversos relatos em que dímeros, trímeros e tetrâmeros apresentam afinidade superior, quando comparados a análogos de maior valência. ${ }^{15-18}$ Esse fato, aliado à simplicidade estrutural e baixo peso molecular dos dímeros, tem despertado o interesse em sua síntese. ${ }^{18-22}$

.Recentemente relatamos a síntese de uma série de ligantes monoméricos derivados de D-galactose e lactose (4- $O$ - $(\beta-\mathrm{D}$ galactopiranosil)-D-glicose) e a interação desses com a lectina de Erythrina cristagalli (LEC). ${ }^{23}$ No presente trabalho é descrita a síntese de dois dímeros derivados de D-galactose, com o mesmo objetivo. Esses dímeros (estruturas 6 e 7, Figura 2) diferem entre si

\footnotetext{
*e-mail: ricardodylan@farmacia.ufmg.br
}

apenas pela presença de uma ligação dupla na cadeia lateral situada na posição quatro da aglicona aromática, resultando em restrição conformacional cuja influência se objetivava avaliar. No planejamento de 6 e 7 levou-se em consideração que a LEC tem maior afinidade por galactosídeos aromáticos de configuração beta. ${ }^{24} \mathrm{~A}$ cadeia lateral com três átomos de carbono, introduzida utilizandose a modificação de Doebner da reação de Knoavenagel, ${ }^{25}$ além de servir como espaçador, permite a variação estrutural pela redução da ligação dupla, gerando derivados com tendências conformacionais diferentes. Ademais, o produto obtido na reação de Doebner apresenta um grupo carboxila terminal, que pode ser convertido em outros grupos funcionais de interesse.

\section{RESULTADOS E DISCUSSÃO}

Inicialmente, a reação do brometo de 2,3,4,6-tetra- $O$-acetil$\alpha$-D-galactopiranosila $(\mathbf{1})^{26}$ com o 4-hidroxibenzaldeído, produto comercial de baixo custo, forneceu o galactosídeo 2 com rendimento de $60 \%$, em escala multigrama. Em seguida, reação de 2 com ácido malônico em presença de piridina ${ }^{25}$ forneceu o derivado cinâmico $\mathbf{3}$ com $75 \%$ de rendimento. A reação de hidrogenação catalítica de $\mathbf{3}$ levou ao derivado hidrogenado 4 com $95 \%$ de rendimento (Figura 1).

Em seguida, a condensação de $\mathbf{3}$ com 1,4-butanodiamina em presença de dicicloexilcarbodi-imida (DCC) e 1-hidroxibenzotriazol $(\mathrm{HOBT})^{27}$ forneceu o dímero peracetilado 5 com $45 \%$ de rendimento. A reação de transesterificação de Zemplén $(\mathrm{MeONa} / \mathrm{MeOH})^{28}$ de 5 forneceu o dímero desprotegido 6 com $99 \%$ de rendimento. A redução catalítica de $\mathbf{6}\left(\mathrm{H}_{2}, \mathrm{Pd}-\mathrm{C}\right)$ forneceu o dímero $7 \mathrm{com} 76 \%$ de rendimento (Figura 2). Alternativamente, o dímero 7 foi obtido pela condensação de $\mathbf{4}$ com a 1,4-butanodiamina, fornecendo o dímero peracetilado correspondente $\mathbf{8}$ com $48 \%$ de rendimento, seguida da desproteção das hidroxilas ( $88 \%$ de rendimento), utilizando-se os mesmos métodos descritos acima para a obtenção de $\mathbf{6}$.

\section{AVALIAÇÃO DA INTERAÇÃO COM A LEC}

O método utilizado para a avaliação dos dímeros 6 e 7 com a LEC foi o ensaio de inibição da hemaglutinação mediada pela 


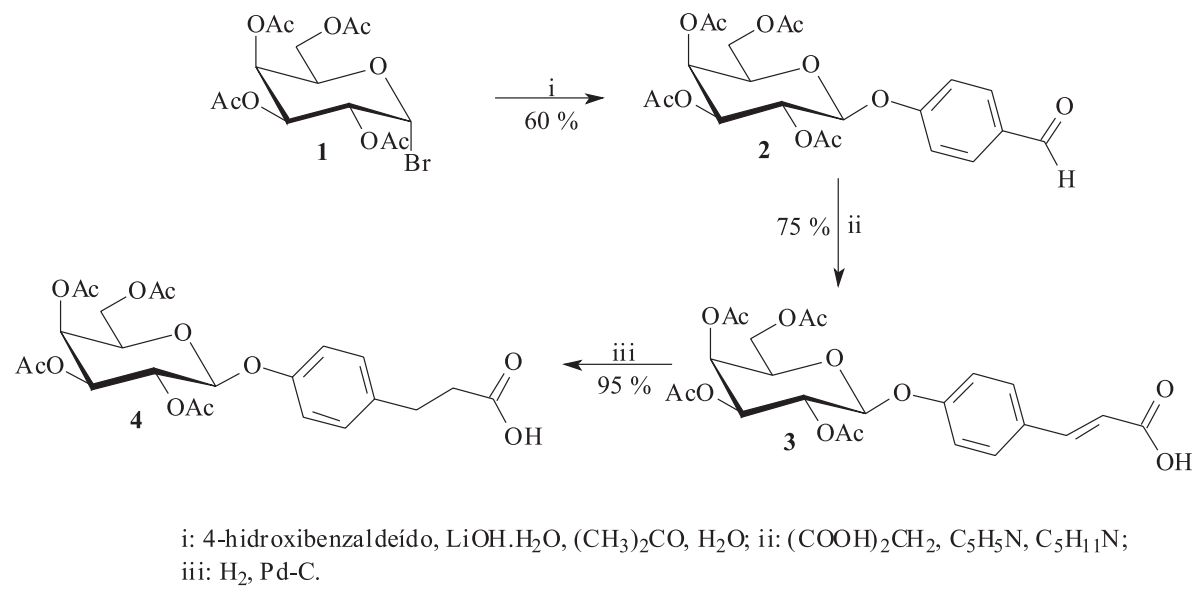

Figura 1. Rota de síntese para obtenção dos galactopiranosídeos intermediários 3 e 4

referida lectina. ${ }^{29}$ Contrariamente ao esperado, o dímero 6 não foi solúvel no meio, impossibilitando sua avaliação e comparação com 7, que apesar de também não ser muito solúvel, foi o suficiente para a avaliação. A potência inibitória de 7 no ensaio foi comparada à da lactose (4-O-( $\beta$-D-galactopiranosil)-D-glicose, dissacarídeo contendo um resíduo de $\mathrm{D}$-galactose de configuração definida (configuração $\beta$ ), cuja potência foi considerada unitária e ao galactosídeo monomérico $\mathbf{9}$, preparado a partir de $\mathbf{4}$ por remoção dos grupos protetores seguida do acoplamento com etanolamina, utilizando-se $N, N$ '-di-isopropilcarbodiimida (DIC) e HOBT como reagentes de acoplamento (Figura 2). Dessa forma, a influência da aglicona pode ser avaliada. Os resultados do ensaio estão apresentados a seguir (Tabela 1).

Tabela 1. Potência relativa de inibição da hemaglutinação mediada por lectina de Erythrina cristagalli pelo dímero 7 e o monômero $\mathbf{9}$, tendo a lactose como referência

\begin{tabular}{lc}
\hline Substância & Potência relativa \\
\hline Lactose & 1,0 \\
7 & 2.7 \\
9 & 1,0 \\
\hline
\end{tabular}

Como pode ser observado, o galactosídeo monomérico 9 apresentou potência relativa equivalente à do dissacarídeo lactose, o que é explicado pelo efeito hidrofóbico da aglicona, devido à interação com um sítio hidrofóbico na lectina. ${ }^{24,30} \mathrm{~A}$ atividade cerca de duas vezes maior do dímero 7 em relação ao ligante monomérico 9 devese ao efeito estatístico (maior concentração local de ligantes). ${ }^{31}$ Pelo fato de a LEC ser uma lectina que apresenta dois sítios de ligação de carboidrato esse resultado era esperado, considerando, é claro, que a natureza e a tendência conformacional do grupo espaçador não causasse perturbação na interação do ligante ao sítio ativo, ${ }^{32}$ o que parece ter sido o caso.

\section{CONCLUSÃO}

Os dímeros 6 e 7 foram sintetizados em quatro e cinco etapas a partir de 4-hidroxibenzaldeído e acetobromogalactose (1), com rendimento global de 20 e $15 \%$, respectivamente. Em ensaio de inibição da hemaglutinação mediada pela lectina de Erythrina cristagalli, o dímero 7 foi 2,7 vezes mais potente do que o ligante monomérico $9 \mathrm{e}$ do que o dissacarídeo lactose, utilizado como composto de referência (potência 1).

\section{PARTE EXPERIMENTAL}

\section{Procedimentos gerais}

As temperaturas de fusão foram determinadas em aparelho Microquímica MQAs 301 e não foram corrigidas. Os valores de poder rotatório específico, $[\alpha]_{\mathrm{D}}$, foram medidos em polarímetros ADP220 Bellinghan + Stanley Ltd. ou Perkin Elmer 341 , a $20^{\circ} \mathrm{C}$. Os espectros de $\mathrm{RMN}$ de ${ }^{1} \mathrm{H}$ e de ${ }^{13} \mathrm{C}$ foram registrados em espectrômetros Bruker Avance DPX-200 ou DRX-400. Como referência interna foi utilizado tetrametilsilano. $\mathrm{CDCl}_{3}, \mathrm{DMSO}-\mathrm{d}_{6}, \mathrm{D}_{2} \mathrm{O}$ ou $\mathrm{CD}_{3} \mathrm{OD}$ foram utilizados como solventes (15-30 mg de amostra por $0,5 \mathrm{~mL}$ de solvente). Os espectros de RMN de ${ }^{1} \mathrm{H}$ e ${ }^{13} \mathrm{C}$ foram obtidos em condições padrão. Os espectros bidimensionais de correlação heteronuclear (HSQC) foram obtidos utilizando sonda de detecção inversa de $5 \mathrm{~mm}$ equipada com gradiente de campo. Os espectros de correlação heteronuclear a longa distância (HMBC) foram obtidos utilizando-se sequência de pulso padrão. Os dados obtidos foram processados em estação de trabalho com programa XWIN-NMR versão 3.1 para Windows Xp.

Os espectros no infravermelho foram registrados em aparelho Spectrum One, Perkin-Elmer. A evolução das reações foi acompanhada por cromatografia em camada delgada de sílica, utilizando-se como revelador solução de ácido sulfúrico $15 \%$ v/v em etanol e aquecimento da placa em estufa. As purificações por cromatografia em coluna de sílica foram realizadas com sílica gel 60, 70-230 mesh (Merck).

\section{Síntese}

2,3,4,6-tetra-O-acetil- $\beta$-D-galactopiranosídeo de 4-formifenila (2)

Dissolveu-se 4-hidroxibenzaldeído (8,11 g, $66 \mathrm{mmol})$ em 19 $\mathrm{mL}$ de solução aquosa de hidróxido de lítio monoidratado (3,02 g, $72 \mathrm{mmol}$ ). Após $10 \mathrm{~min}$, foram adicionados $28 \mathrm{~mL}$ de solução de brometo de 2,3,4,6-tetra- $O$-acetil- $\alpha$-D-galactopiranosila (12) $(8,85 \mathrm{~g}$, $21 \mathrm{mmol})$ em acetona. A mistura foi mantida sob agitação magnética, à temperatura ambiente, por $1 \mathrm{~h}$. Após a remoção do solvente, a mistura obtida foi dissolvida em uma mistura de diclorometano e água e transferida para funil de separação. As duas fases foram separadas e a fase orgânica foi extraída com solução de hidróxido de sódio 0,5 mol.L $\mathrm{L}^{-1}$ (4 X $50 \mathrm{~mL}$ ) e, em seguida, com água destilada até $\mathrm{pH}$ 7. A fase orgânica foi secada com sulfato de sódio anidro, filtrada e concentrada em evaporador rotatório.

O galactosídeo $2(5,82 \mathrm{~g}, 12,9 \mathrm{mmol})$ foi isolado com $60 \%$ de rendimento.

Sólido branco. F. F. $111-115^{\circ} \mathrm{C}$ (literatura $\left.121-122{ }^{\circ} \mathrm{C}\right){ }^{33}[\alpha]_{\mathrm{D}}$ $-5,3\left(\mathrm{c} 2, \mathrm{CHCl}_{3}\right)\left(\right.$ literatura $[\alpha]_{\mathrm{D}}-1,1\left(\mathrm{c} 1, \mathrm{CH}_{3} \mathrm{OH}\right)^{29}$. IR $\bar{v}_{\max }\left(\mathrm{cm}^{-1}\right)$ : 


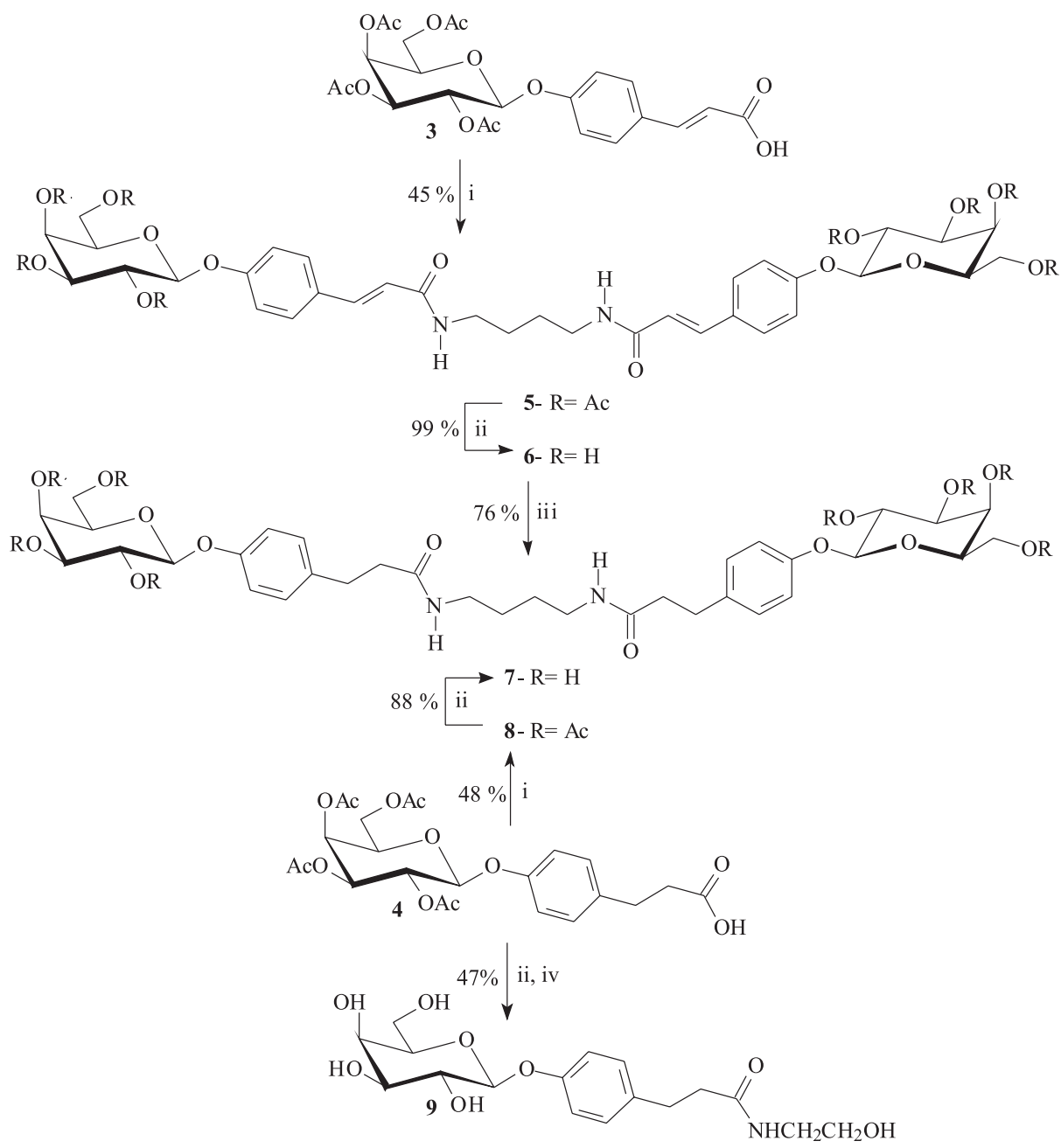

i: DCC, $\mathrm{HOBT}, \mathrm{NH}_{2} \mathrm{CH}_{2} \mathrm{CH}_{2} \mathrm{NH}_{2}$, DMF, $\mathrm{CH}_{2} \mathrm{Cl}_{2}$; ii: $\mathrm{MeONa} / \mathrm{MeOH}$; iii: $\mathrm{H}_{2}$, Pd-C; iv: DIC, $\mathrm{HOBT}, \mathrm{NH}_{2} \mathrm{CH}_{2} \mathrm{CH}_{2} \mathrm{OH}$, DMF.

Figura 2. Rota de síntese para obtenção dos dímeros 6 e 7 e do monômero 9

1736, 1699, 1602, 1579, 1376, 1220, 1044, 824. $\mathrm{RMN}$ de $\mathrm{H}^{1}\left(\mathrm{CDCl}_{3}\right.$; $200 \mathrm{MHz}$ ): 9,93 (s; 1H; CㅂO); 7,86 (d; $J=8,44,2 \mathrm{H}$; arom.); 7,12 (d; $J=8,44,2 \mathrm{H}$; arom.); 5,58-5,48 (m; 2H; H-1 e H-4); 5,20-5,11 (m; 2H; H-2 e H-3); 4,24-4,09 (m; 3H; H-5, H-6 e H-6'); 2, 19-2,03 (s; $\left.12 \mathrm{H} ; \mathrm{COC}_{3}\right)$. RMN de $\left.{ }^{13} \mathrm{C}\left(\mathrm{CDCl}_{3}, 50 \mathrm{MHz}\right): 190,72 \underline{\mathrm{CHO}}\right)$; $170,34-169,32\left(\underline{\mathrm{COCH}}_{3}\right) ; 161,29\left(\mathrm{C}_{i p s o}\right) ; 131,83\left(2 \mathrm{C}_{\text {arom. }} \mathrm{e}_{i p s o}\right) ; 116,76$ $\left(2 \mathrm{C}_{\text {arom }}\right) ; 98,61(\mathrm{C}-1) ; 71,35(\mathrm{C}-3$ ou C-5); 70,68 (C-5 ou C-3); 68,40 (C-2); 66,76 (C-4); 61,37 (C-6); 20,71-20,57 (CO댁)

\section{2,3,4,6-tetra-O-acetil- $\beta$-D-galactopiranosídeo de 4-(E)-}

(carboxietenil)fenila $(\mathbf{3})$

O galactosídeo 2 (1,0 g, 2,21 mmol) e ácido malônico (1,3 g, 12,5 mmol) foram adicionados a uma solução constituída de $3,3 \mathrm{~mL}$ de piridina anidra e $0,25 \mathrm{~mL}$ de piperidina. A mistura reagente foi mantida sob agitação, a $50{ }^{\circ} \mathrm{C}$ por $1 \mathrm{~h}$ e, em seguida, a $110^{\circ} \mathrm{C}$ por $2 \mathrm{~h}$. Em seguida, a mistura de reação foi vertida em gelo pilado e ácido clorídrico concentrado foi acrescentado, gota a gota, até $\mathrm{pH} 1$. O derivado cinâmico 3 foi obtido após filtração $(0,9 \mathrm{~g}, 1,82 \mathrm{mmol})$ com $75 \%$ de rendimento.

Sólido branco. F. F. $76,8-77,2{ }^{\circ}$ C. $[\alpha]_{D}-6,3\left(\mathrm{c} 1, \mathrm{CHCl}_{3}\right)$, IR $\overline{\mathrm{v}}_{\max }$ $\left(\mathrm{cm}^{-1}\right)$ : 1743, 1686, 1632, 1603, 1509, 1427, 1211, 1041. RMN de $\mathrm{H}^{1}\left(\mathrm{CDCl}_{3} ; 200 \mathrm{MHz}\right): 7,74(\mathrm{~d} ; J=15,92,1 \mathrm{H}) ; 7,51(\mathrm{~d} ; J=8,70,2 \mathrm{H}$; arom.); 7,02 (d; $J=8,70,2 \mathrm{H}$; arom.); 6,36 (d; $J=15,92,1 \mathrm{H}) ; 5,51$ (dd; $J=10,55 ; 7,82 ; 1 \mathrm{H} ; \mathrm{H}-2) ; 5,47$ (d; $J=3,55 ; 1 \mathrm{H} ; \mathrm{H}-4) ; 5,13$ (dd; $J=$
$10,55 ; 3,40 ; 1 \mathrm{H} ; \mathrm{H}-3) ; 5,11$ (d; $J=7,91 ; 1 \mathrm{H} ; \mathrm{H}-1) ; .2,22-2,02(\mathrm{~s} ; 12 \mathrm{H}$; $\left.\mathrm{COC} \underline{H}_{3}\right)$. RMN de ${ }^{13} \mathrm{C}\left(\mathrm{CDCl}_{3}, 50 \mathrm{MHz}\right): 171,80(\underline{\mathrm{COOH}}) ; 170,40-$ $169,40\left(\underline{C O C H}_{3}\right) ; 158,61\left(\mathrm{C}_{i p s o}\right) ; 146,02(\mathrm{HC}=\underline{C} \mathrm{H}-\mathrm{COOH}) ; 129,96$ $\left(2 \mathrm{C}_{\text {arom }}\right) ; 129,21\left(\mathrm{C}_{i p s o}\right) ; 117,07\left(2 \mathrm{C}_{\text {arom }}\right) ; 116,12(\mathrm{H} \underline{C}=\mathrm{CH}-\mathrm{COOH})$; 99,02 (C-1); 71,18 (C-3 ou C-5); 70,75 (C-5 ou C-3); 68,51(C-2); 66,81 (C-4); 61,36 (C-6); 20,73-20,58 (COC $\left.\mathrm{H}_{3}\right)$.

\section{2,3,4,6-tetra-O-acetil- $\beta$-D-galactopiranosídeo de 4-(carboxietil) fenila (4)}

O galactosídeo 3 (200 mg, 0,40 mmol) foi dissolvido em $10 \mathrm{~mL}$ de metanol. À solução, foram adicionados $150 \mathrm{mg}$ de Pd/C e a mistura reagente foi mantida sob agitação em hidrogenador Parr a 25 psi de gás hidrogênio até consumo do material de partida, observado por CCD. O catalisador foi removido por filtração e o solvente removido em evaporador rotatório, fornecendo 4 (191 mg, 0,39 mmol) com 95\% de rendimento.

Sólido branco. F. F. $189-192^{\circ} \mathrm{C}$. $[\alpha]_{\mathrm{D}}+2,4\left(\mathrm{c} 0,8, \mathrm{CHCl}_{3}\right)$; IR $\overline{\mathrm{v}}_{\max }$ ( $\left.\mathrm{cm}^{-1}\right): 2937,1742,1212,1042,914 . \mathrm{RMN} \mathrm{de} \mathrm{H}^{1}\left(\mathrm{CDCl}_{3} ; 200 \mathrm{MHz}\right)$ : 7,14 (d; $J=8,53 ; 2 \mathrm{H}$; arom.); 6,93 (d; $J=8,53,2 \mathrm{H}$; arom.); 5,52-5,43 (m; 2H; H-2 e H-4); 5, 11 (dd; $J=10,49 ; 3,33 ; 1 \mathrm{H} ; \mathrm{H}-3) ; 5,02$ (d; $J=$ $7,92 ; 1 \mathrm{H} ; \mathrm{H}-1) ; 2,92$ (t; $\left.J=7,47 ; 2 \mathrm{H} ; \mathrm{PhCH}_{2} \underline{\mathrm{H}}_{2}\right) ; 2,65$ (t; $J=7,47$; $\left.2 \mathrm{H} ; \mathrm{PhC}_{2} \mathrm{CH}_{2}\right) ; 2,19-1,86\left(\mathrm{~s} ; 12 \mathrm{H} ; \mathrm{COC} \underline{H}_{3}\right) . \mathrm{RMN} \mathrm{de}{ }^{13} \mathrm{C}\left(\mathrm{CDCl}_{3}\right.$, $50 \mathrm{MHz}): 178,16(\underline{\mathrm{COOH}}) ; 170,64-169,65\left(\underline{\mathrm{COCH}}_{3}\right) ; 155,64\left(\mathrm{C}_{i p s o}\right)$; 
$135,35\left(\mathrm{C}_{\mathrm{ipso}}\right) ; 129,53\left(2 \mathrm{C}_{\text {arom }}\right) ; 117,24\left(2 \mathrm{C}_{\text {arom }}\right) ; 99,92(\mathrm{C}-1) ; 71,10$ (C-5 ou C-3); 71,02 (C-3 ou C-5); 68,85 (C-2); 67,08 (C-4); 61,54 (C-6); 35,77 $\left(\mathrm{PhCH}_{2} \underline{\mathrm{C}} \mathrm{H}_{2}\right) ; 29,95\left(\mathrm{Ph}_{\underline{C}} \mathrm{CH}_{2}\right) ; 20,89-20,75\left(\mathrm{CO} \underline{\mathrm{C}} \mathrm{H}_{3}\right)$.

$N, N$-bis-[4-(2,3,4,6-tetra-O-acetil- $\beta$-D-galactopiranosiloxi)-(E)benzenopropenoil]-1,4- butanodiamina (5)

A $5 \mathrm{~mL}$ de uma mistura 2:1 de diclorometano: $N, N$-dimetilformamida foram adicionados $600 \mathrm{mg}(1,21 \mathrm{mmol})$ de $3,250 \mathrm{mg}(1,21$ mmol) de dicicloexilcarbodi-imida (DCC) e $163 \mathrm{mg}(1,21 \mathrm{mmol})$ de 1-hidrobenzotriazol (HOBT). A mistura reagente foi mantida sob agitação a $0{ }^{\circ} \mathrm{C}$, sob atmosfera de nitrogênio. Após $10 \mathrm{~min}$, uma solução de 1,4-butanodiamina (40 mg, 0,45 mmol), dissolvida em $2 \mathrm{~mL}$ de diclorometano, foi adicionada. A mistura reagente foi mantida sob agitação magnética, à temperatura ambiente, por $14 \mathrm{~h}$. A dicicloexilureia formada foi removida por filtração e o solvente foi removido em evaporador rotatório. O resíduo obtido $(623 \mathrm{mg})$ foi submetido à cromatografia em coluna de sílica. $\mathrm{O}$ dímero 5 foi obtido das frações eluídas com acetato de etila:metanol 1:1 com $45 \%$ de rendimento $(210 \mathrm{mg}, 0,20 \mathrm{mmol})$.

Sólido branco, F.F. $189-192{ }^{\circ} \mathrm{C} .[\alpha]_{\mathrm{D}}+11,5\left(\mathrm{c} 0,2,\left(\mathrm{CH}_{3}\right)_{2} \mathrm{CO}\right)$; IR $\bar{v}_{\text {max }}\left(\mathrm{cm}^{-1}\right): 1742,1655,1605,1509,1367,1215,1043,828 . \mathrm{RMN}$ de $\mathrm{H}^{1}\left(\mathrm{CDCl}_{3} ; 400 \mathrm{MHz}\right): 7,58(\mathrm{~d} ; 1 \mathrm{H} ; J=15,40 ; \mathrm{PhCH}=\mathrm{C} \underline{H}) ; 7,48-$ 7,44 (m; 2H; arom.); 6,99-6,95 (m; 2H; arom.); 6,39-6,35 (m; $1 \mathrm{H}$; $\mathrm{PhC} \underline{H}=\mathrm{CH}$ ); 5,51-5,46 (m; 2H; H-2 e H-4); 5, 15-5,08 (m; 2H; H-1 e H-3); 4,23-4,09 (m; 3H; H-5, H-6 e H-6'); 3,34 (s; $\mathrm{NHC}_{2} \mathrm{CH}_{2}$ ); 1,62 $\left(\mathrm{s} ; \mathrm{NHCH}_{2} \underline{\mathrm{CH}}_{2}\right) ; 2,18-1,97\left(\mathrm{~s} ; 12 \mathrm{H} ; \mathrm{COCH}_{3}\right) . \mathrm{RMN} \mathrm{de}{ }^{13} \mathrm{C}\left(\mathrm{CDCl}_{3}\right.$, $100 \mathrm{MHz}): 172,18-171,46\left(\underline{\mathrm{COCH}}_{3}\right) ; 168,96(\underline{\mathrm{CONH}}) ; 159,57\left(\mathrm{C}_{i p s o}\right)$; $140,99(\mathrm{HC}=\underline{C} \mathrm{H}-\mathrm{CONH}) ; 131,43\left(\mathrm{C}_{i p s o}\right) ; 130,48\left(2 \mathrm{C}_{\text {arom }}\right) ; 120,90$ $(\mathrm{H} \underline{C}=\mathrm{CH}-\mathrm{CONH}) ; 118,16\left(\mathrm{C}_{\text {arom }}\right) ;$ 99,91 (C-1); 73,69 (C-3 ou C-5); 72,41 (C-5 ou C-3); 70,33 (C-2); 68,89 (C-4); 62,75 (C-6); 40,36 $\left.\left(\mathrm{HN}_{\mathrm{C}} \mathrm{H}_{2} \mathrm{CH}_{2}\right) ; 28,08\left(\mathrm{HNCH}_{2} \underline{\mathrm{H}}_{2}\right) ; 20,75-20,63(\mathrm{CO} \underline{\mathrm{CH}})_{3}\right)$

\section{$N, N$ '-bis-[4-( $\beta$-D-galactopiranosiloxi)-(E)-benzenopropenoil]-1,4- butanodiamina $(\mathbf{6})$}

Uma solução de metóxido de sódio foi preparada pela dissolução de $0,8 \mathrm{~g}$ de sódio em $10 \mathrm{~mL}$ de metanol anidro. A solução foi resfriada a $-5{ }^{\circ} \mathrm{C}$ e, então, $600 \mathrm{mg}(0,58 \mathrm{mmol})$ do dímero 5 foram adicionados. A mistura de reação foi mantida sob agitação magnética até consumo total do material de partida, observado por CCD. Foi acrescentado metanol suficiente para dissolver o produto gelatinoso formado e foi acrescentada quantidade suficiente de resina Amberlite IRA 120 para neutralização. Após filtração e evaporação do solvente, o dímero 6 foi obtido (402 mg, $0.57 \mathrm{mmol}$ ) com $99 \%$ de rendimento.

Sólido branco, F. F.151-153 ${ }^{\circ} \mathrm{C} .[\alpha]_{\mathrm{D}}+5,3$ (c 0,4, DMSO); IR $\overline{\mathrm{v}}_{\max }\left(\mathrm{cm}^{-1}\right): 3322,1655,1601,1508,1226,1043,831 . \mathrm{RMN}$ de ${ }^{1} \mathrm{H}$ (DMSO- $\left.d_{6} ; 200 \mathrm{MHz}\right): 7,49$ (d; $J=8,74,2 \mathrm{H}$; arom.); 7,36 (d; $1 \mathrm{H} ; J$ $=15,70 ; \mathrm{PhCH}=\mathrm{C} \underline{H}) ; 7,04$ (d; $J=8,74,2 \mathrm{H} ;$ arom. $) ; 6,48(\mathrm{~d} ; 1 \mathrm{H} ; J=$ 15,70; $\mathrm{PhC} \underline{H}=\mathrm{CH}) ; 5,21-4,52$ (m; 5H; H-2, H-3, H-5, H-6 e H-6'); $4,86(\mathrm{~d} ; J=7,50 ; 1 \mathrm{H} ; \mathrm{H}-1) ; 3,34\left(\mathrm{~d} ; J=4,12 ; \mathrm{NHC}_{2} \mathrm{CH}_{2}\right) ; 1,47$ (s; $\left.\mathrm{NHCH}_{2} \mathrm{CH}_{2}\right) ; \mathrm{RMN}$ de ${ }^{13} \mathrm{C}$ (DMSO- $\left.d_{6}, 50 \mathrm{MHz}\right): 165,21(\underline{\mathrm{CONH}})$; $158,45\left(C_{i p s o}\right), 138,13(\mathrm{HC}=\underline{C} \mathrm{H}-\mathrm{CONH}) ; 128,93\left(C_{i p s o}\right) ; 128,60\left(2 \mathrm{C}_{\mathrm{a}}\right.$ )$; 120,34(\mathrm{H} \underline{C}=\mathrm{CH}-\mathrm{CONH}) ; 116,58\left(2 \mathrm{C}_{\text {arom }}\right) ; 100,74(\mathrm{C}-1) ; 75,59$ (C-3 ou C-5); 73,30 (C-5 ou C-3); 70,28 (C-2); 68,18 (C-4); 60,42 (C-6); 38,47 ( $\left.\mathrm{HNC}_{2} \mathrm{CH}_{2}\right) ; 26,85\left(\mathrm{HNCH}_{2} \underline{\mathrm{C}} \mathrm{H}_{2}\right)$.

\section{$N, N$ '-bis-[4-(2,3,4,6-tetra-O-acetil- $\beta$-D-galactopiranosiloxi) benzenopropanoil]-1,4-butanodiamina $(\mathbf{8})$}

O dímero protegido 8 foi preparado com $48 \%$ de rendimento a partir de 4 (900 mg, 1,81 mmol), DCC (560 mg, 2,72 mmol), HOBT (367 mg, 2,72 mmol) e 1,4-butanodiamina (34 mg, 0,39 mmol), por caminho sintético essencialmente semelhante ao descrito para preparação de 5 . Ao final da reação, o solvente foi removido em evaporador rotatório e o resíduo $(1,15 \mathrm{~g})$ foi submetido à cromatografia em coluna de sílica, fornecendo $8 \mathrm{com} 48 \%$ de rendimento (359 $\mathrm{mg}, 0,34 \mathrm{mmol}$ ) após eluição com acetato de etila:metanol.

Sólido branco. F. F.. $155-156^{\circ} \mathrm{C}$. $[\alpha]_{\mathrm{D}}-9,4\left(\mathrm{c} 0,6, \mathrm{CHCl}_{3}\right)$; IR $\bar{v}_{\text {max }}$ $\left(\mathrm{cm}^{-1}\right)$ : 1739, 1643, 1534, 1214, 1042, 828. RMN de $\mathrm{H}^{1}\left(\mathrm{CDCl}_{3} ; 400\right.$ MHz): 7,21 (d; $J=8,32 ; 2 \mathrm{H}$; arom.); 6,91 (d; $J=8,32 ; 2 \mathrm{H}$; arom.); 5,99 (s; 1H; NH); 5,48-5,45 (m; 2H; H-2 e H-4); 5, 12 (dd; $J=10,48$; 3,36; 1H; H-3); 5,02 (d; $J=7,96 ; 1 \mathrm{H} ; \mathrm{H}-1$ ); 4,21 (dd; $J=11,18 ; 6,60$; 1H; H-6); 4,15 (dd; $J=11,18 ; 6,60 ; 1 \mathrm{H} ; \mathrm{H}-6$ '); 4,06 (t; $J=6,60 ; 1 \mathrm{H}$; $\mathrm{H}-5) ; 3,15$ (m; $\left.\mathrm{NHC}_{2} \mathrm{CH}_{2}\right) ; 2,93\left(\mathrm{t} ; J=7,34 ; 1 \mathrm{H} ; \mathrm{PhC}_{2} \underline{\mathrm{CH}}_{2}\right) ; 2,48$ $\left(\mathrm{t} ; J=7,34 ; 1 \mathrm{H} ; \mathrm{PhCH}_{2} \underline{C}_{2}\right) ; 1,32\left(\mathrm{~m} ; \mathrm{NHCH}_{2} \underline{\mathrm{H}}_{2}\right) ; 2,16-2,01$ (s; $\left.12 \mathrm{H} ; \mathrm{COCH}_{3}\right) . \mathrm{RMN}$ de ${ }^{13} \mathrm{C}\left(\mathrm{CDCl}_{3}, 100 \mathrm{MHz}\right): 172,26(\underline{\mathrm{CONH}})$; $170,31-169,36\left(\underline{C O C H}_{3}\right) ; 155,40\left(\mathrm{C}_{i p s o}\right) ; 135,86\left(\mathrm{C}_{i p s o}\right) ; 129,52\left(2 \mathrm{C}_{\mathrm{a}-}\right.$ $\left.{ }^{2}\right) ; 117,03\left(\mathrm{C}_{\text {arom }}\right)$; 99,91 (C-1); 70,99 (C-5 ou C-3); 70,76 (C-3 ou C-5); 68,69 (C-2); 66,88 (C-4); 61,26 (C-6); 39,01 ( $\left.\mathrm{NHCH}_{2} \mathrm{CH}_{2}\right)$; $38,40\left(\mathrm{PhCH}_{2} \underline{\mathrm{C}} \mathrm{H}_{2}\right) ; 30,92\left(\mathrm{Ph}_{\underline{C}} \mathrm{H}_{2} \mathrm{CH}_{2}\right) ; 26,61\left(\mathrm{NHCH}_{2} \underline{\mathrm{C}} \mathrm{H}_{2}\right) ; 20,69-$ $20,53\left(\mathrm{CO}_{\mathbf{C}}{ }_{3}\right)$.

\section{$N, N$ '-bis-[4-( $\beta$-D-galactopiranosiloxi)benzenopropanoil]-1,4- butanodiamina $(\mathbf{7})$ \\ A partir de 6}

O dímero 7 foi obtido com $76 \%$ de rendimento ( $76 \mathrm{mg}, 0,11$ mmol) a partir de 6 (100 mg, 0,14 mmol) e $60 \mathrm{mg} \mathrm{de} \mathrm{Pd} / \mathrm{C}$, nas condições descritas para a preparação de 4 .

\section{A partir de 8}

O dímero 7 foi obtido com $88 \%$ de rendimento $(350 \mathrm{mg}, 0,57$ mmol), pela reação de 8 (590 mg, 0,56 mmol) com metóxido de sódio/metano, como descrito para 6 .

Sólido branco. F.F. $110-111^{\circ} \mathrm{C} .[\alpha]_{\mathrm{D}}-3,16$ (c 0,6, DMSO); IR $\bar{v}_{\max }\left(\mathrm{cm}^{-1}\right): 3300,2946,1613,1557,1239,1074,831 . \mathrm{RMN}$ de $\mathrm{H}^{1}$ (CD $\left.{ }_{3} \mathrm{OD} ; 400 \mathrm{MHz}\right): 7,12$ (d; $J=8,56,2 \mathrm{H}$; arom.); 7,01 (d; $J=8,56$, $2 \mathrm{H}$; arom.); 4,89-4,80 (m; $2 \mathrm{H}$; H-1 e H-3); 3,90 (d; $J=3,30 ; 1 \mathrm{H}$; H-4); 3,82-3,72 (m; 3H; H-2, H-6 e H-6') 3,57 (dd; $J=9,72 ; 3,30$, $1 \mathrm{H} ; \mathrm{H}-3) ; 3,66(\mathrm{t} ; J=6,07 ; 1 \mathrm{H} ; \mathrm{H}-5) ; 3,06\left(\mathrm{~m} ; \mathrm{NHC}_{2} \mathrm{CH}_{2}\right) ; 2,85(\mathrm{t}$; $\left.J=7,43 ; 2 \mathrm{H} ; \mathrm{PhCH}_{2} \underline{\mathrm{CH}}_{2}\right) ; 2,43\left(\mathrm{t} ; J=7,43 ; 2 \mathrm{H} ; \mathrm{PhC}_{\underline{H}_{2}} \mathrm{CH}_{2}\right) ; 1,28$ (s; $\left.\mathrm{NHCH}_{2} \mathrm{C}_{2}\right)$; $\mathrm{RMN} \mathrm{de}{ }^{13} \mathrm{C}\left(\mathrm{CD}_{3} \mathrm{OD}, 100 \mathrm{MHz}\right): 175,34$ ( $\left.\underline{\mathrm{CONH}}\right)$; $157,84\left(\mathrm{C}_{i p s o}\right) ; 136,12\left(\mathrm{C}_{i p s o}\right) ; 130,52\left(2 \mathrm{C}_{\text {arom }}\right) ; 117,98\left(2 \mathrm{C}_{\text {arom }}\right) ; 103,27$ (C-1); 77,06 (C-3 ou C-5); 75,03 (C-5 ou C-3); 72,48 (C-2); 70,35 (C-4); 62,56 (C-6); 40,10 (NHCH$\left.{ }_{2} \mathrm{CH}_{2}\right) ; 39,27\left(\mathrm{PhCH}_{2} \mathrm{CH}_{2}\right) ; 32,27$ $\left(\mathrm{Ph}_{\mathrm{C}} \mathrm{H}_{2} \mathrm{CH}_{2}\right) ; 27,71\left(\mathrm{NHCH}_{2} \underline{\mathrm{CH}}_{2}\right)$.

$N$-(2-hidroxietil)-4-( $\beta$-D-galactopiranosiloxi)benzenopropanamida (9)

Inicialmente, o galactosídeo $4(611 \mathrm{mg}, 1,23 \mathrm{mmol})$ foi submetido à metanólise, conforme descrito para $\mathbf{6}$, fornecendo o derivado desprotegido correspondente (384 mg, 1,17 mmol) com 95\% de rendimento. Este foi transferido para balão de $10 \mathrm{~mL}$, utilizando-se $5 \mathrm{~mL}$ de $N, N$-dimetilformamida anidra. Em seguida, foram acrescentados $301 \mathrm{mg}$ de 1-hidroxibenzotriazol (2,23 mmol), $350 \mu \mathrm{L}$ de diisopropilcarbodi-imida (2,23 mmol) e, então, $81 \mu \mathrm{L}$ de 2 -aminoetanol $(1,34 \mathrm{mmol})$. A mistura reagente foi mantida sob agitação a $60{ }^{\circ} \mathrm{C}$. Após o término da reação, verificado por CCD (aproximadamente $12 \mathrm{~h}$ ), o solvente foi removido em evaporador rotatório e a mistura obtida foi tratada com acetona e metanol. Foram obtidos $206 \mathrm{mg}$ ( $0,16 \mathrm{mmol})$ da amida 9 , com $47 \%$ de rendimento.

Sólido branco. F.F. $178-180^{\circ}$ C. $[\alpha]_{D}-3,1$ (c 0,8 , DMSO). RMN de ${ }^{1} \mathrm{H}\left(\mathrm{D}_{2} \mathrm{O} ; 300 \mathrm{MHz}\right): 7,13$ (d, $J_{9,8}$ 7,7 Hz, H-9 e H-9'), 6,99 (d, $J_{8,9} 7,7$ Hz, H-8 e H-8'), 4,93 (d, J 6,2 ,6 Hz, H-1), 3,91-3,68 (m, H-2, H-3, H-4, H-5, H-6 e H-6'), 3,42 (t, $J_{15,14} 4,8$ Hz, H-15), 3, 12 (t, $J_{14,15} 4,8$ Hz, H-14), 2,80 (t, $\left.J_{11,12} 6,7 \mathrm{~Hz}, \mathrm{H}-12\right), 2,44$ (t, $J_{12,11} 6,7 \mathrm{~Hz}, \mathrm{H}-11$ ). $\mathrm{RMN}$ de ${ }^{13} \mathrm{C}\left(\mathrm{D}_{2} \mathrm{O} ; 75 \mathrm{MHz}\right): 172,3(\underline{\mathrm{CONH}}) ; 156,2$ (C-7); 135,0 (C-10); 129,5 (C-9); 116,6 (C-8); 100,9 (C-1); 75,3 (C-5 ou C-3); 72,5 (C-3 ou C-5); 70,5 (C-2); 68,4 (C-4); 60,7 (C-6); 59,8 (C-15); 41,1 (C-14); 37,6 (C-12); 30,6 (C-11). 


\section{MATERIAL SUPLEMENTAR}

No Material Suplementar, disponível em http://quimicanova.sbq. org.br, na forma de arquivo PDF, com acesso livre, estão incluídos os espectros de $\mathrm{RMN}{ }^{1} \mathrm{H}$ e $\mathrm{RMN}{ }^{13} \mathrm{C}$ das substâncias 2-8, os mapas de contornos HMQC de $\mathbf{3}$ e 8, o mapa de contornos COSY e expansão da região de $\delta 2$ a $\delta 6$ ppm de $\mathbf{8}$.

\section{AGRADECIMENTOS}

Ao CNPq, CAPES e FAPEMIG a concessão de auxílio financeiro, bolsa de doutorado (R. C. Figueiredo) e bolsa de produtividade (M. A. F. Prado e R. J. Alves)

\section{REFERÊNCIAS}

1. Imberty, A.; Varrob, A.; Curr. Opin. Struct. Biol. 2008, 18, 567.

2. Dwek, R. A.; Chem. Rev. 1996, 96, 683.

3. Cloninger, M. J.; Curr. Opin. Chem. Biol. 2002, 6, 2.

4. Bezouska, K.; Rev. Mol. Biotechnol. 2002, 90, 269.

5. Kim, Y.; Zimmerman, S. C.; Curr. Opin. Chem. Biol. 1998, 2, 733.

6. Bosman, A. W.; Janssen, H. M.; Chem. Rev. 1999, 99, 1665.

7. Toone, E. J.; Curr. Opin. Struct. Biol. 1994, 4, 719.

8. Kiessling, L. L.; Pohl, N. L.; Chem. Biol. 1996, 3, 71.

9. Lieth, C.; Grank.; M., Lindhorst, T.; Rev. Mol. Biotechnol. 2002, 90, 311.

10. Roy, R.; Kim, J. M.; Tetrahedron 2003, 59, 3881.

11. Han, S.; Baigude, H.; Hattori, K.; Yoshida, T.; Uryu, T.; Carbohydr. Polym. 2007, 68, 26.

12. Roy, R.; Trends Glycosci. Glycotechnol. 2203, 15, 291.

13. Lee, Y. C.; Lee, R. T.; Acc. Chem. Res. 1995, 28, 321.

14. Lundquist, J. J.; Toone, E. J.; Chem. Rev. 2002, 102, 555.

15. Krist, P.; Vannuci, L.; Kuzma, M.; Man, P.; Sadalapure, K.; Patel, A.; Bezouska, K.; Pospisil, M.; Petrus, L.; Lindhorst, T. K.; Krem, V.; ChemBioChem. 2004, 5, 445.

16. Baek, M. G.; Roy, R.; Bioorg. Med. Chem. 2002, 10, 11.
17. Lee, R. T.; Lee, Y. C.; Glyconjugate 2000, 17, 543.

18. Maierhofer, C.; Rohmer, K.; Wittmann, V.; Bioorg. Med. Chem. Lett. 2007, 15, 7661 .

19. Roy, R.; Das, S. K.; Dominique, R.; Trono, M. C.; Hernández-Mateo, F.; Santoyo-González, F.; Pure Appl. Chem. 1999, 71, 565.

20. Roy, R.; Das, S. K.; Hernández-Mateo, F.; Santoyo-Gonzáles, F.; Gan, Z.; Synthesis 2001, 1049.

21. Hayes, W.; Osborn, H. M. I.; Osborne, S. D.; Rastall, R. A.; Romagnoli, B.; Tetrahedron 2003, 59, 7983.

22. Ahmad, N.; Gabius, H-J.; Sabesan, S.; Oscarson, S.; Brewer, C. F.; Glycobiology 2004, 14, 817.

23. Butera, A. P.; Souza Filho, J. D.; Carvalho, D. T.; Figueiredo, R. C.; Faria, L. C. A.; Nunes, M. A.; Prado, M. A. F.; Alves, R. J.; Andrade, M. H. G.; Silva, K. T. S.; Quim. Nova 2007, 30, 1267.

24. Liener, I. V.; Sharon, N.; Goldstein, I. J.; The Lectins. Properties, Functions and Applications in Biology and Medicine, Academic Press: Orlando, 1986.

25. Percec, V.; Peterca, M.; Sienkowska, M. J.; Ilies, M. A.; Aqad, E.; Smidrkal, J.; Heiney, P. A.; J. Am. Chem. Soc. 2006, 128, 3324.

26. Kozikowski, A. P.; Lee, J.; J. Org. Chem. 1990, 55, 863.

27. Ashton, P. R.; Boyd, S. E.; Brown, C. L.; Jayaraman, N.; Nepogodiev, N.; Stoddart, J. F.; Chem. Eur. J. 1996, 2, 1115.

28. Misra, A. K.; Tiwari, P.; Madhusudan, S. K.; Carbohydr. Res. 2005, 340, 325.

29. Knozy, E. H.; Bernardes, E. S.; Rosa, C.; Faça, V.; Greene, L. J.; Ward, R. J.; Arch. Biochem. Biophys. 2003, 410, 222.

30. Davis, B. G.; J. Chem. Soc., Perkin Trans. 1 1999, 3215.

31. Gestwicki, J. E.; Cairo, C. W.; Strong, L. E.; Oetjen, K. A.; Kiessling, L. L.; J. Am. Chem. Soc. 2002, 124, 14922.

32. André, S.; Frisch, B.; Kaltner, H.; Desouza, D. L.; Schuber, F.; Gabius, H-J.; Pharm. Res. 2000, 17, 985.

33. Olsufyeva, E. N.; Tevyashova, A. N.; Trestchalin, I. D.; Preobrazhenskaya, M. N.; Platt, D.; Klyosov, A.; Carbohydr. Res. 2003, 338, 1359. 


\section{SÍNTESE DE $\beta$-D-GALACTOPIRANOSÍDEOS DE ARILA DIMÉRICOS PARA AVALIAÇÃO DE SUA INTERAÇÃO COM A LECTINA DE Erythrina cristagalli}

Rute Cunha Figueiredo, Nádia Burkowski Meyer, Maria Auxiliadôra Fontes Prado e Ricardo José Alves*

Departamento de Produtos Farmacêuticos, Faculdade de Farmácia, Universidade Federal de Minas Gerais, Av. Antônio Carlos, 6627, 31270-901 Belo Horizonte - MG, Brasil

Javier Rojo

Grupo de Carbohidratos, Instituto de Investigaciones Químicas, CSIC, Américo Vespucio 49, E-41092 Sevilla, Espanha

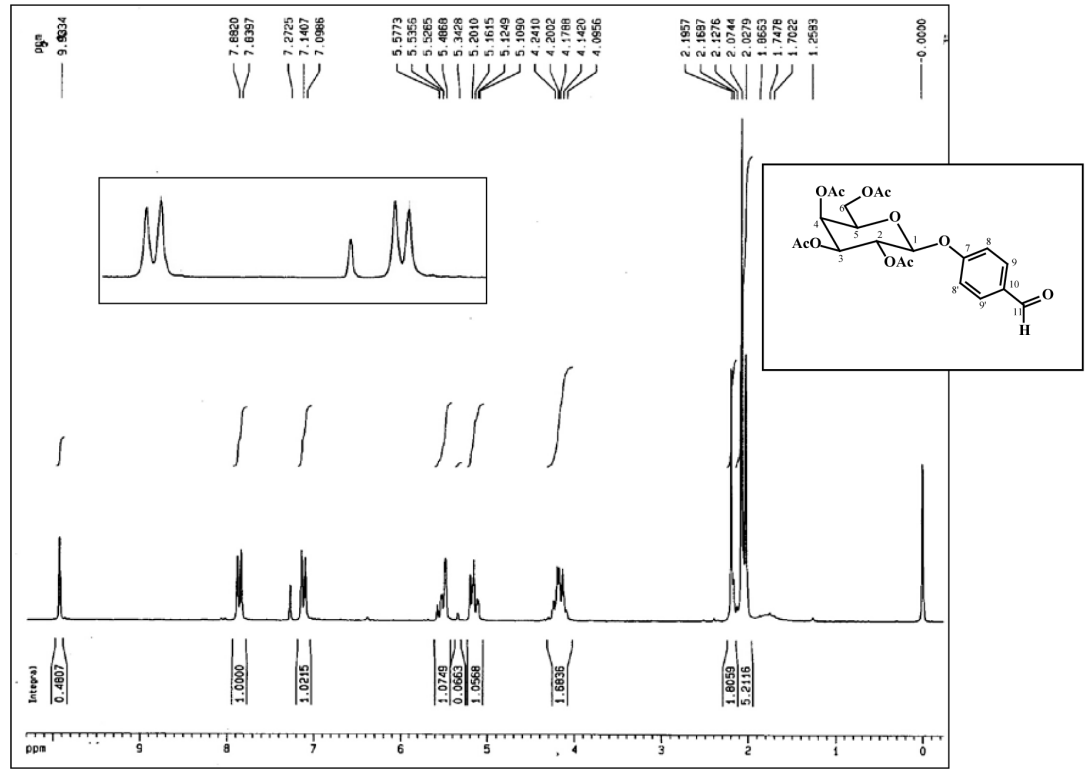

Figura 1Sa. Espectro de $\mathrm{RMN}$ de ${ }^{\mathrm{l}} \mathrm{H}$ de $2\left(\mathrm{CDCl}_{3}, 200 \mathrm{MHz}\right)$

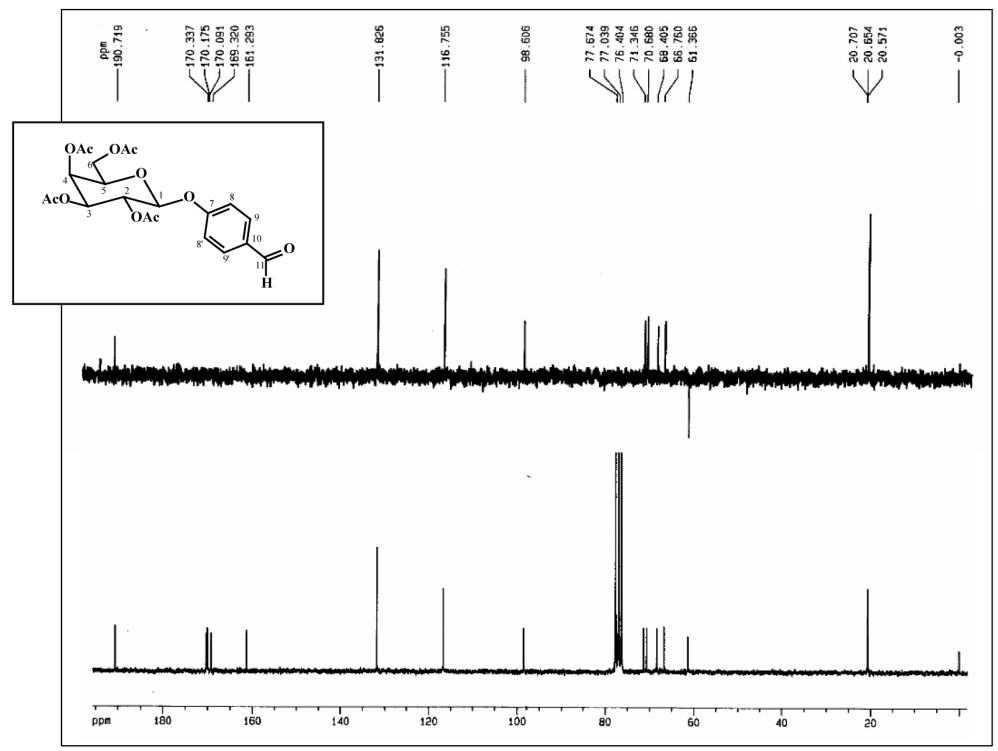

Figura 1Sb. Espectro de RMN de ${ }^{13} \mathrm{C}$ e subespectro DEPT 135 de $2\left(\mathrm{CDCl}_{3}, 50 \mathrm{MHz}\right)$

*e-mail: ricardodylan@farmacia.ufmg.br 


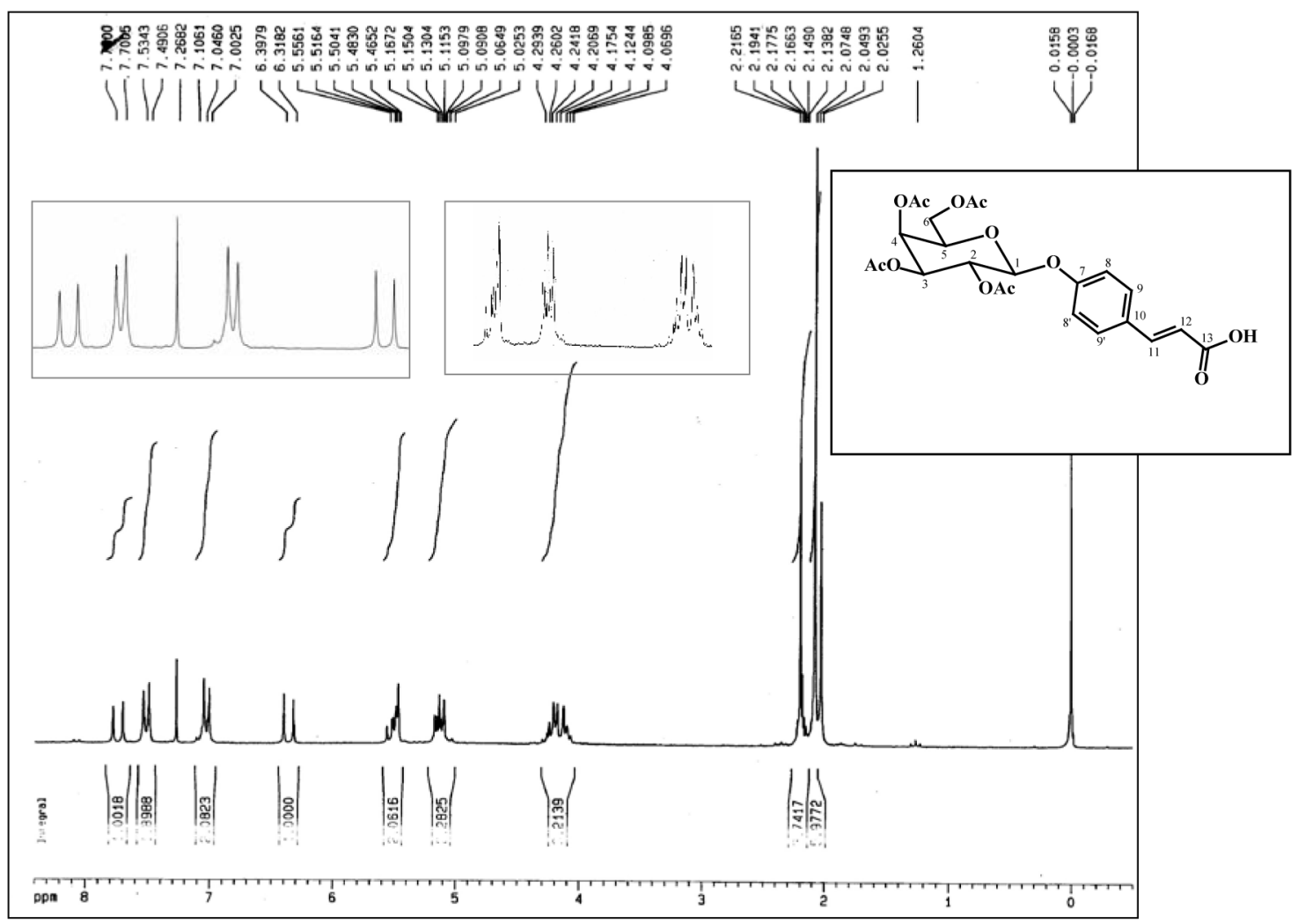

Figura 2Sa. Espectro de $\mathrm{RMN}$ de ${ }^{l} \mathrm{H}$ de $\mathbf{3}\left(\mathrm{CDCl}_{3}, 200 \mathrm{MHz}\right)$

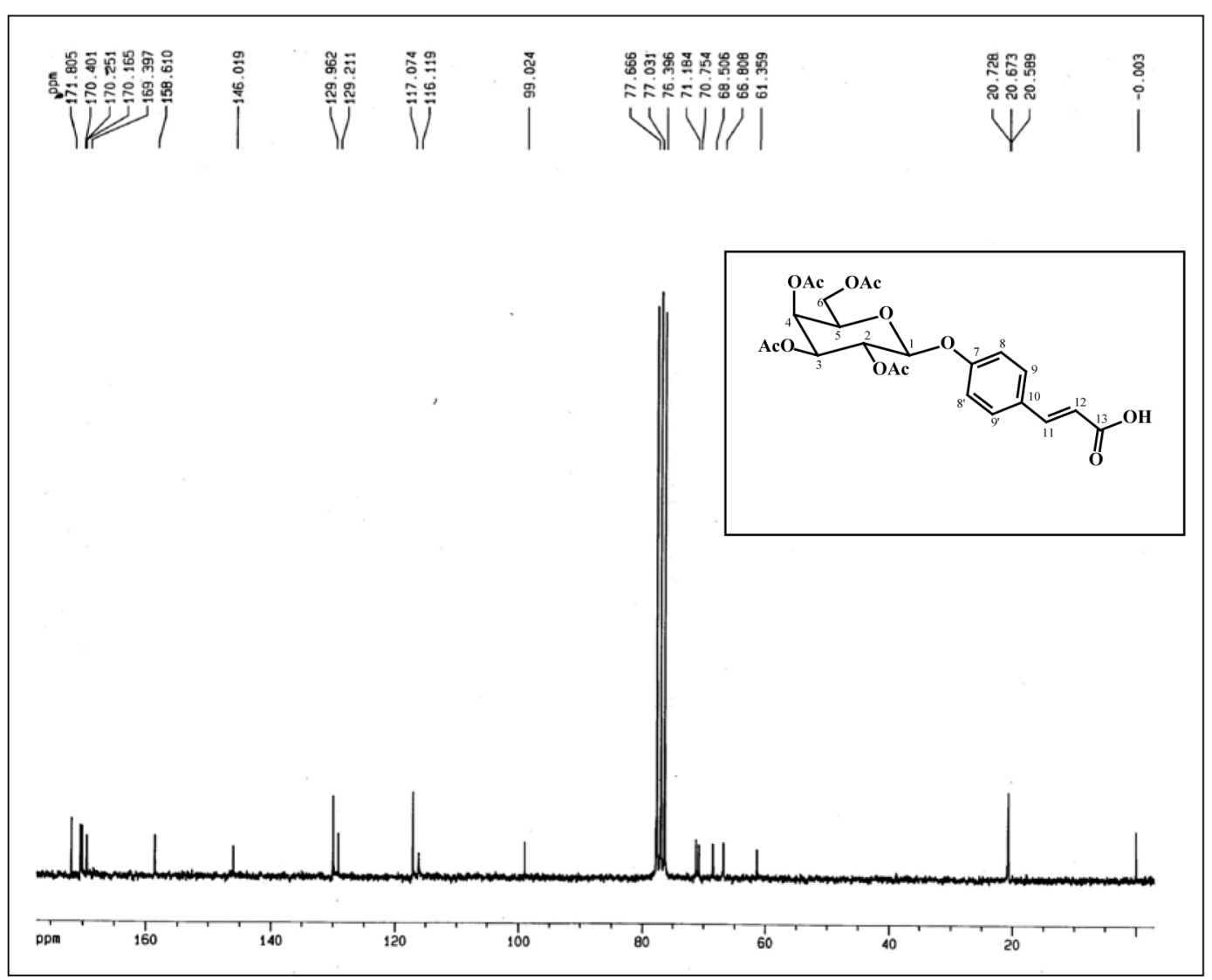

Figura 2Sb. Espectro de $\mathrm{RMN} \mathrm{de}{ }^{13} \mathrm{C}$ de $3\left(\mathrm{CDCl}_{3}, 50 \mathrm{MHz}\right)$ 


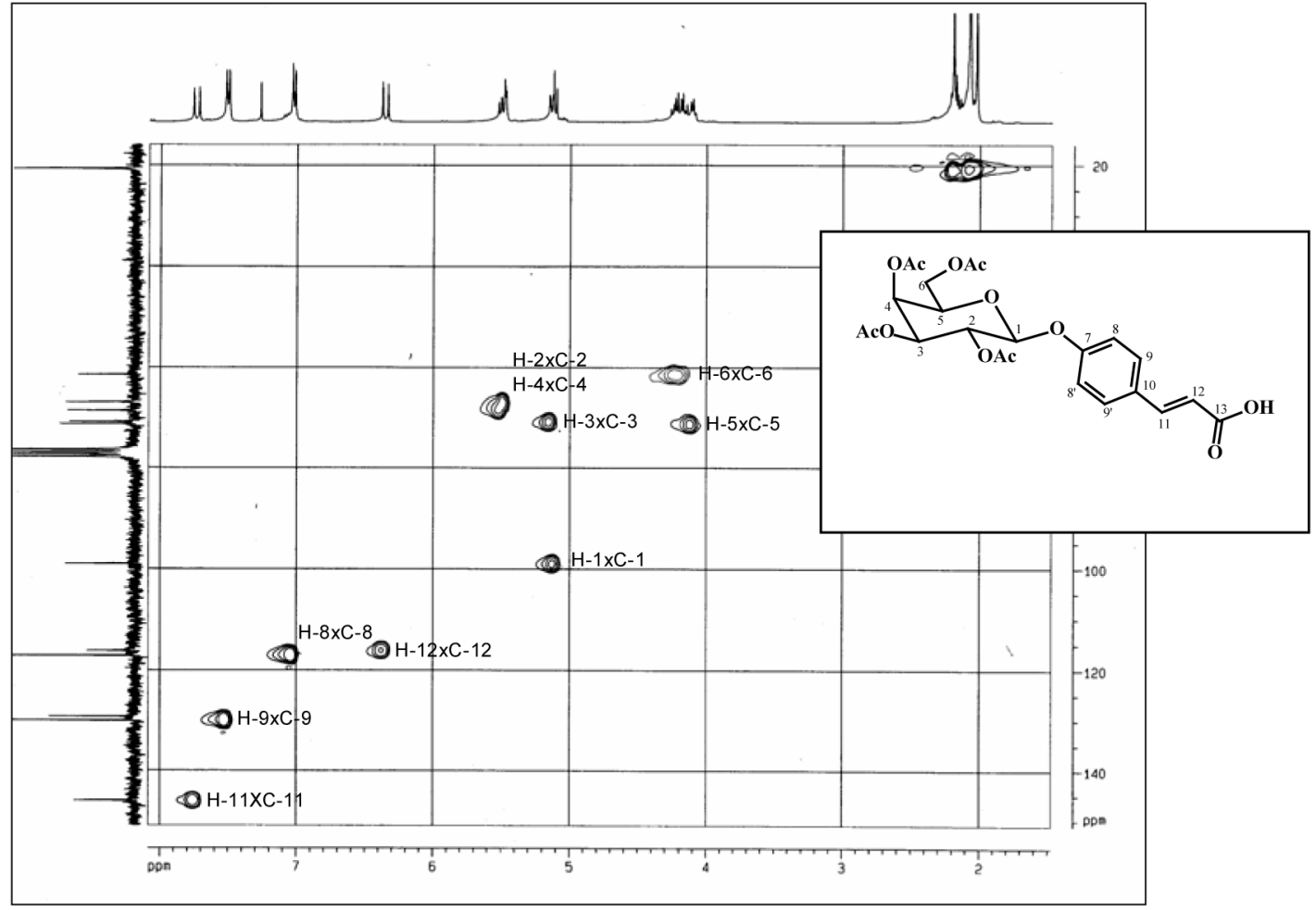

Figura 3Sa. Mapa de contornos HMQC de $3\left(\mathrm{CDCl}_{3}, 400 \mathrm{MHz}\right)$

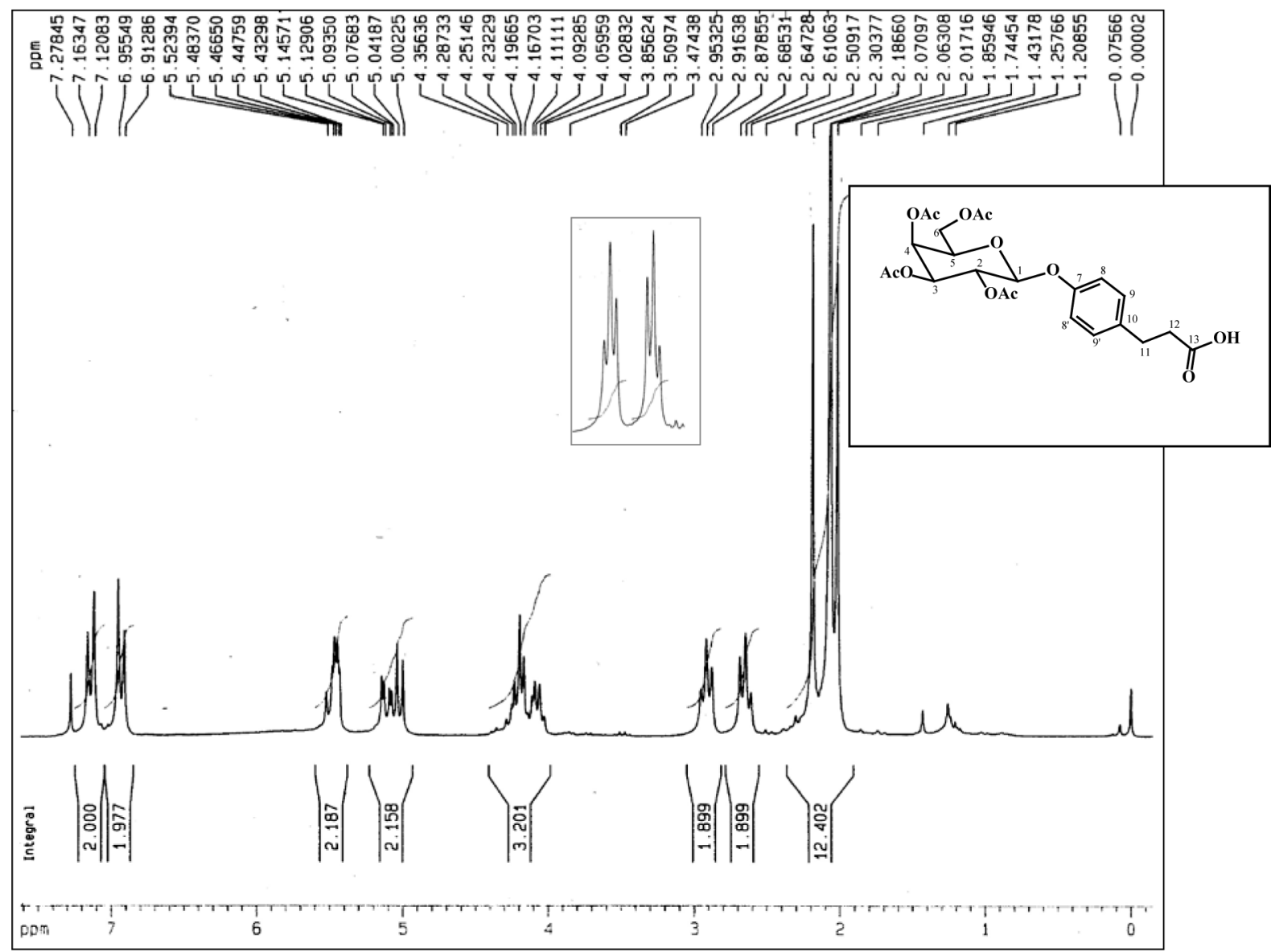

Figura 3Sb. Espectro de $\mathrm{RMN}$ de ${ }^{l} \mathrm{H}$ de $4\left(\mathrm{CDCl}_{3}, 200 \mathrm{MHz}\right)$ 


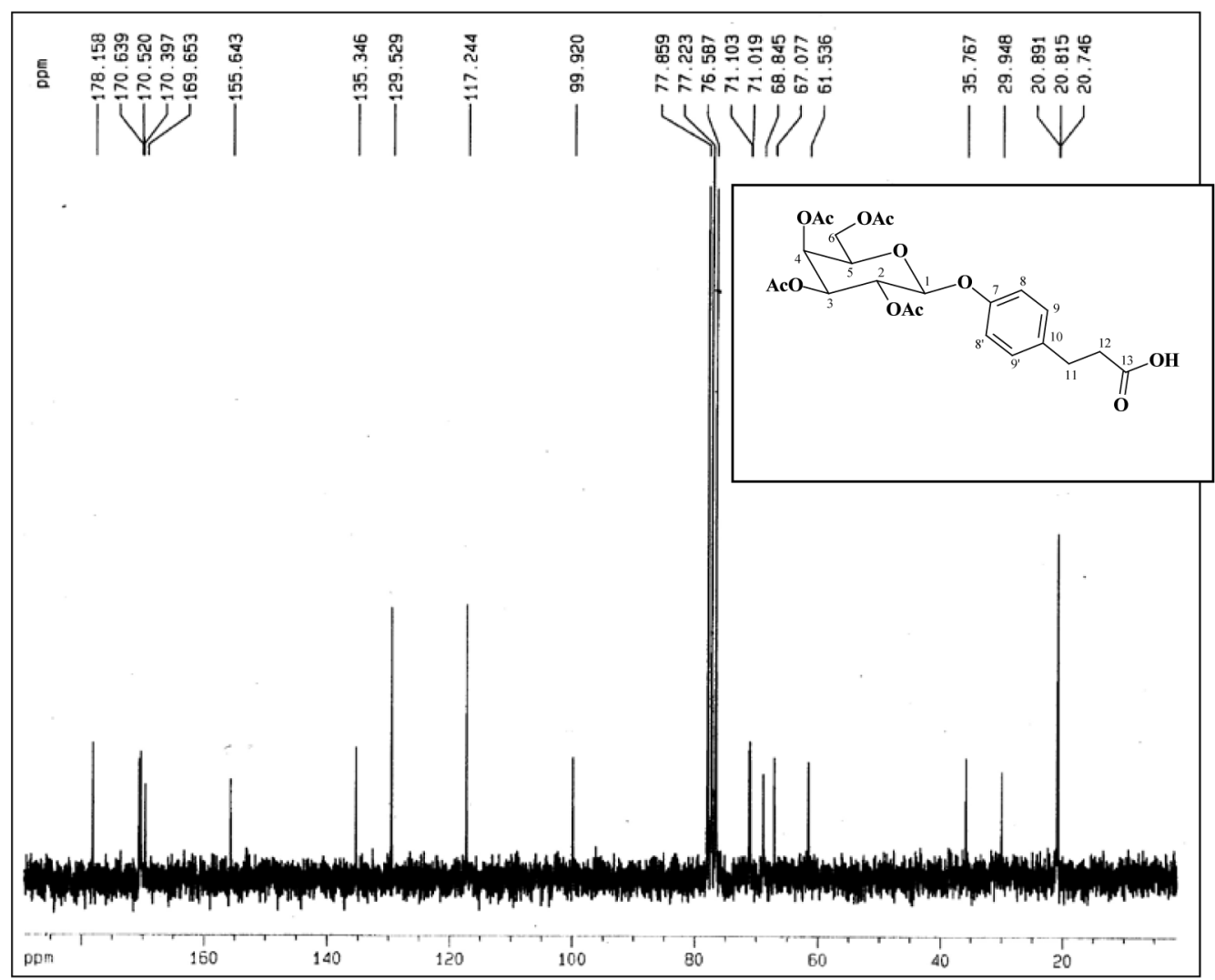

Figura 4Sa. Espectro de $\mathrm{RMN} \mathrm{de}^{13} \mathrm{C}$ de $4\left(\mathrm{CDCl}_{3}, 50 \mathrm{MHz}\right)$

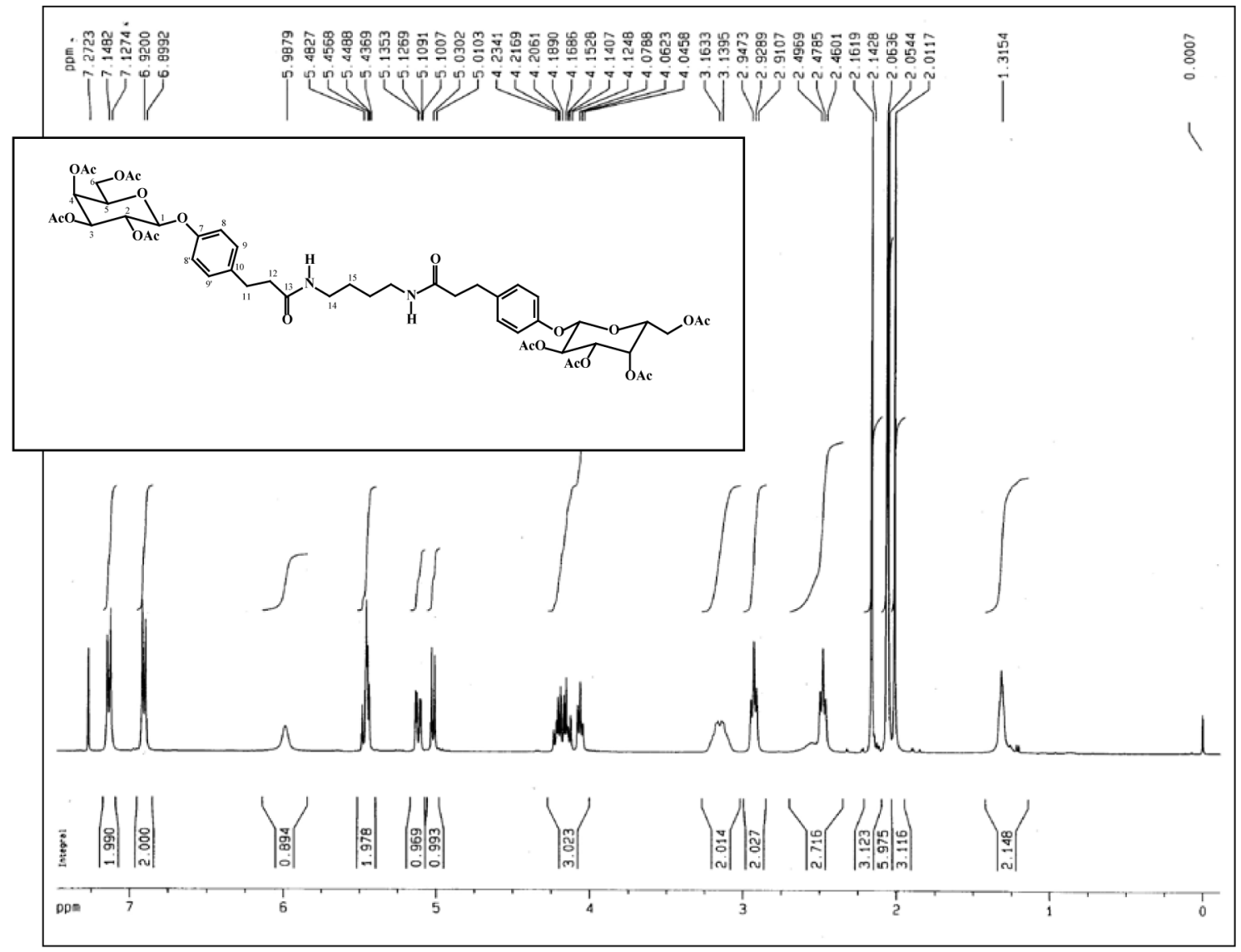

Figura 4Sb. Espectro de $\mathrm{RMN}$ de ${ }^{1} \mathrm{H}$ de $8\left(\mathrm{CDCl}_{3}, 400 \mathrm{MHz}\right)$ 


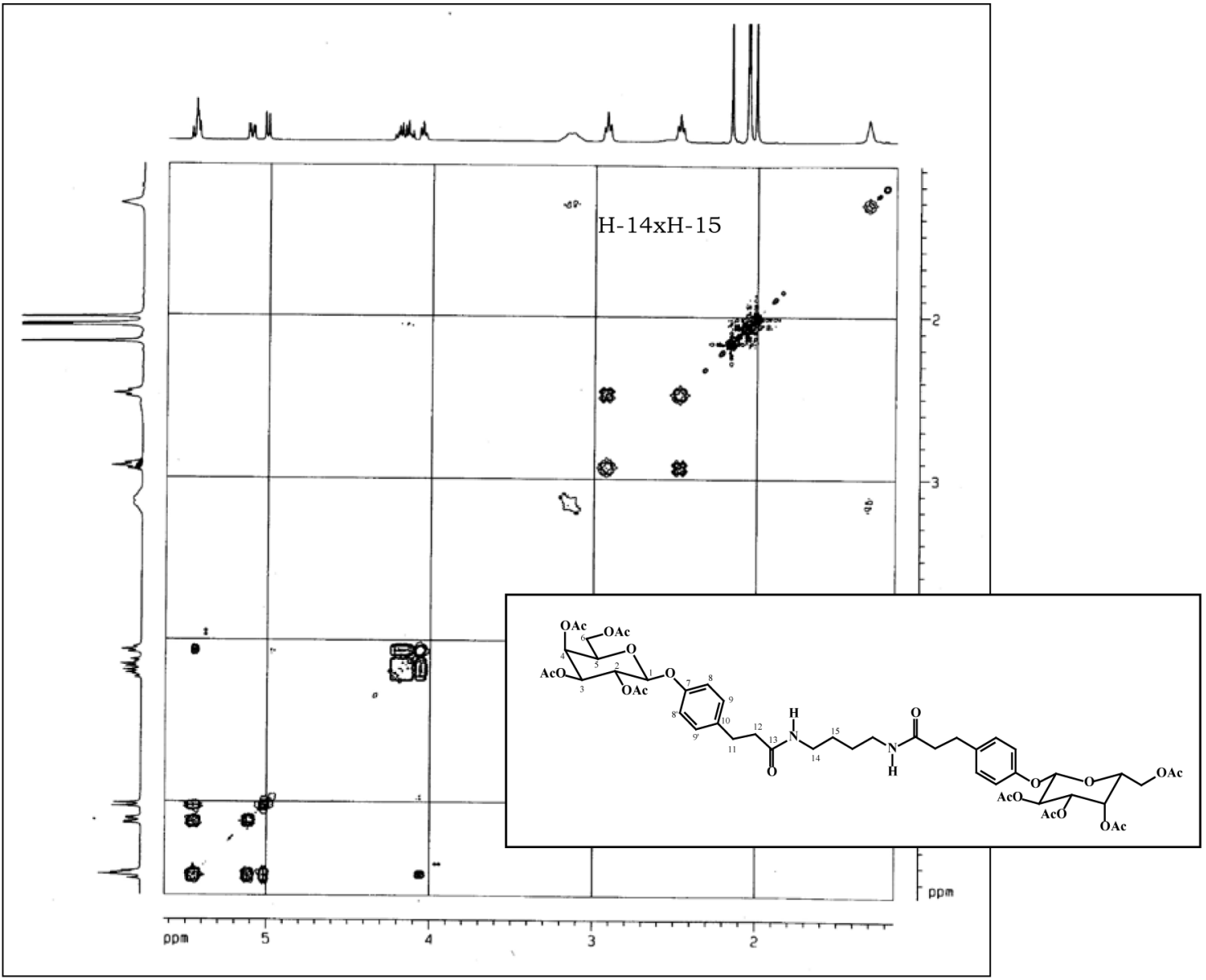

Figura 5Sa. Mapa de contornos COSY de $8\left(\mathrm{CDCl}_{3}, 400 \mathrm{MHz}\right)$

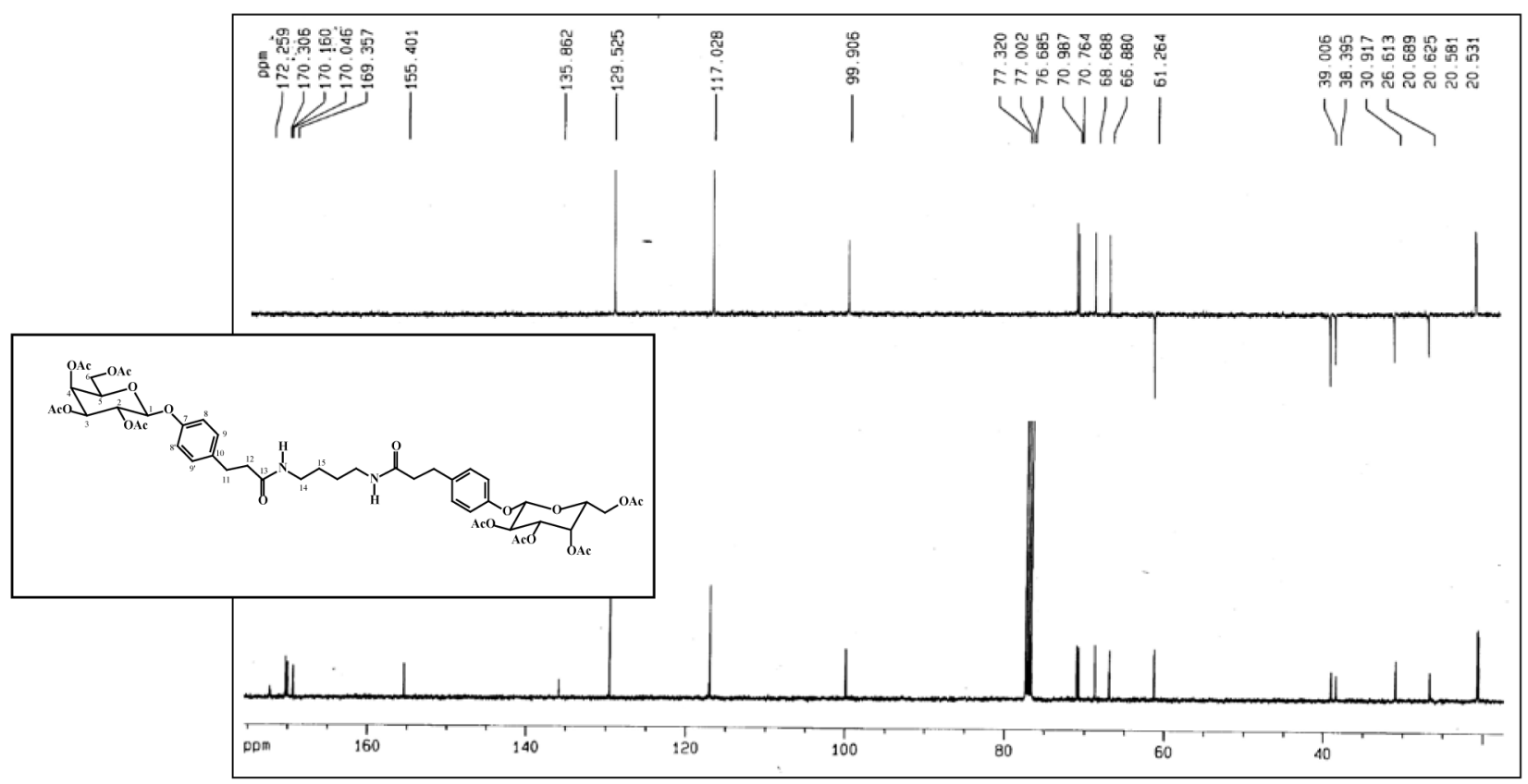

Figura 5Sb. Espectro de RMN de ${ }^{13} \mathrm{C}$ e subespectro DEPT 135 de $8\left(\mathrm{CDCl}_{3}, 100 \mathrm{MHz}\right)$ 


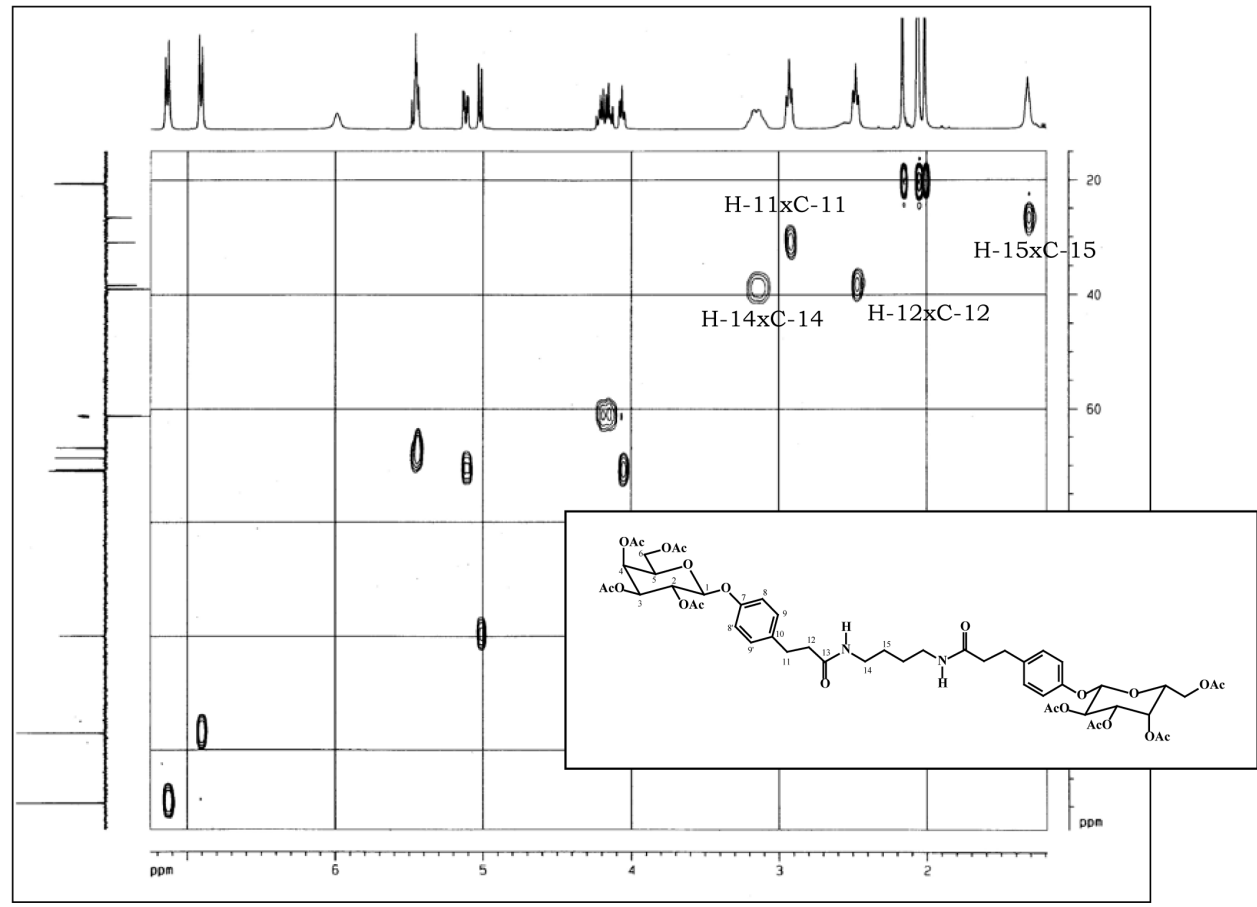

Figura 6S. Mapa de contornos $\mathrm{HMQC} \mathrm{de} 8\left(\mathrm{CDCl}_{3}, 100 \mathrm{MHz}\right)$

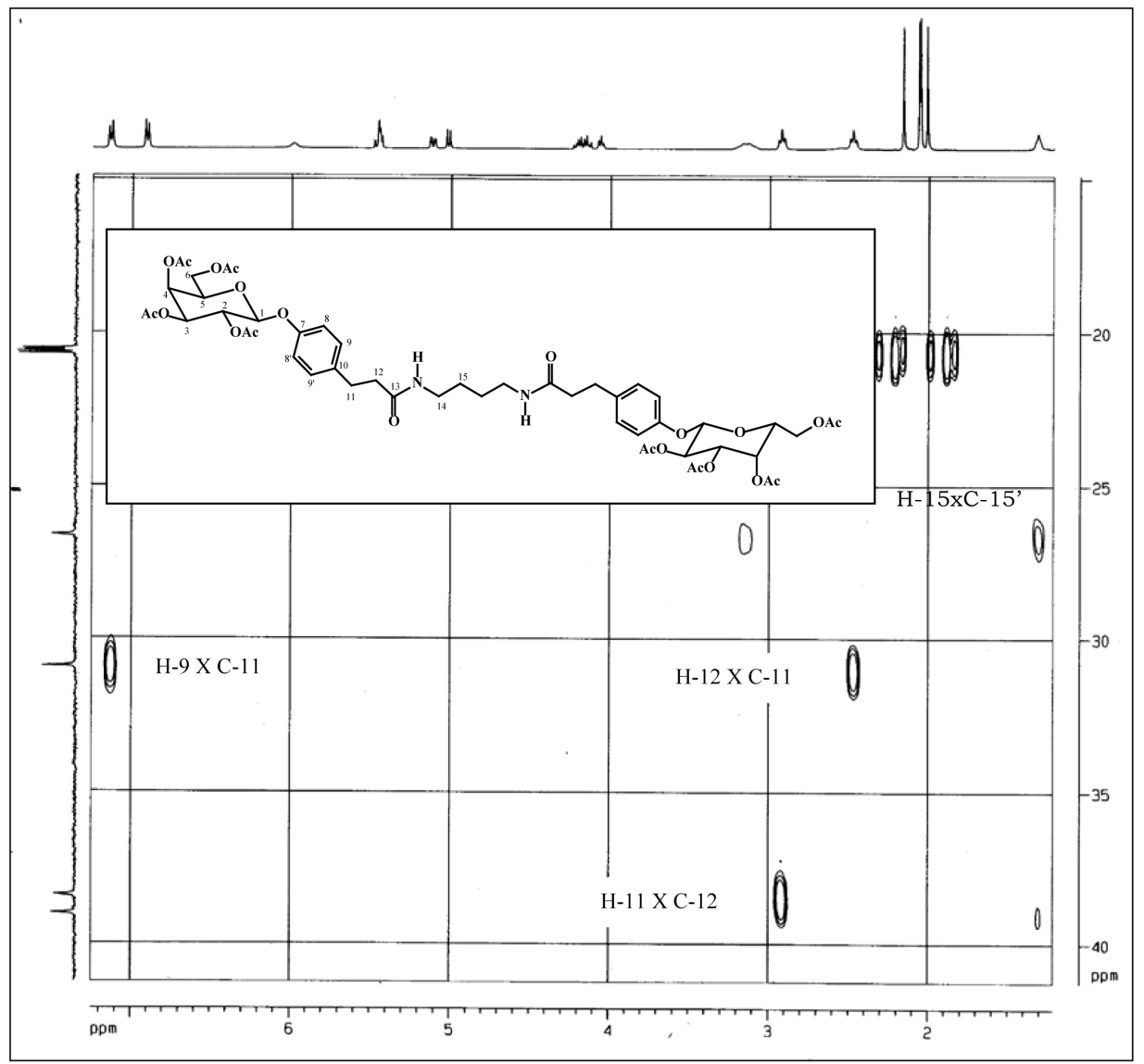

Figura 7S. Ampliação do mapa de contornos $H M B C$ de 8 


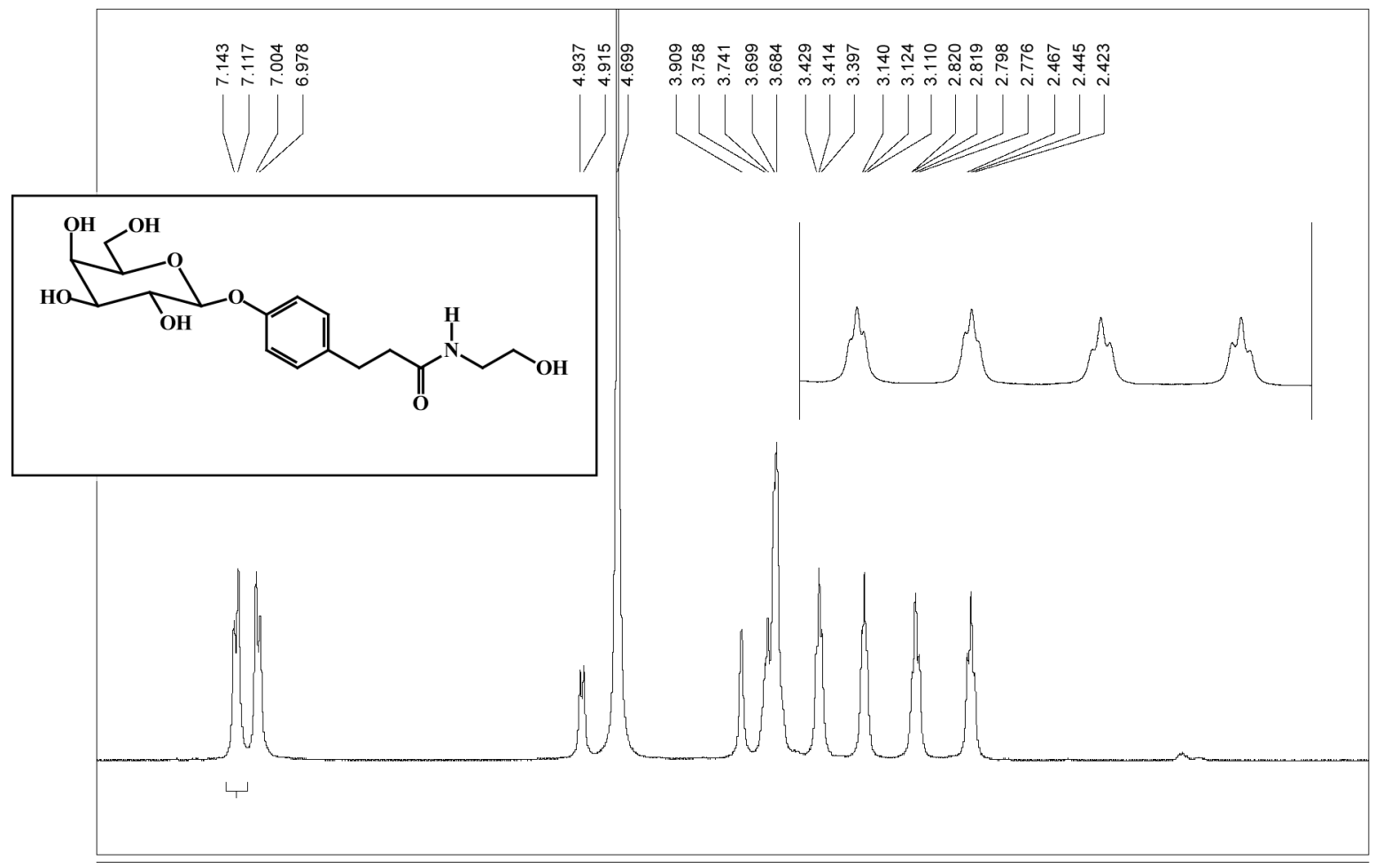

Figura 8Sa. Espectro $\mathrm{RMN}$ de ${ }^{l} \mathrm{H}$ de $9\left(\mathrm{D}_{2} \mathrm{O}, 300 \mathrm{MHz}\right)$

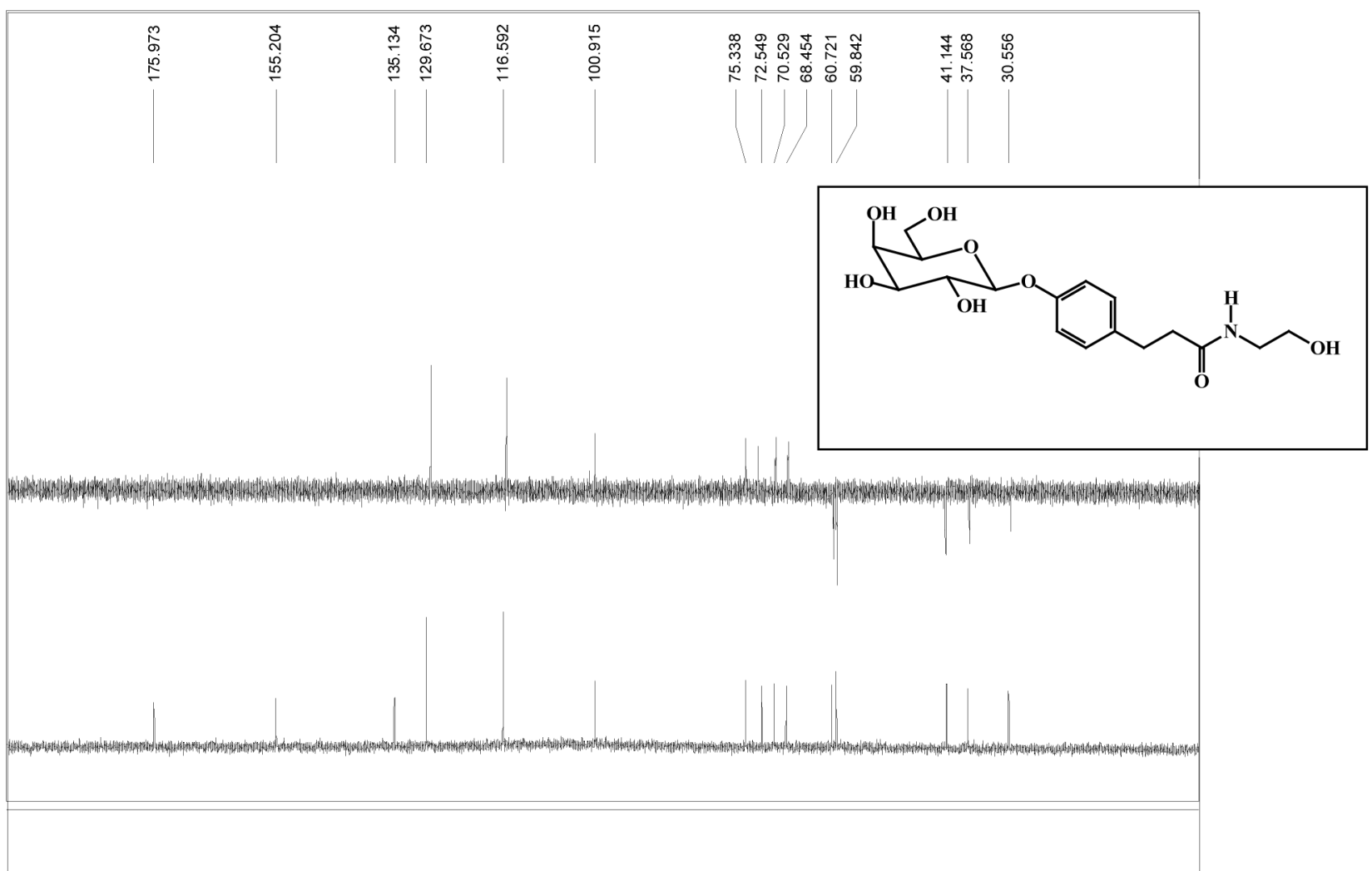

Figura 8Sb. Espectro de $\mathrm{RMN}$ de ${ }^{13} \mathrm{C}$ de $9\left(\mathrm{D}_{2} \mathrm{O}, 75 \mathrm{MHz}\right)$ 


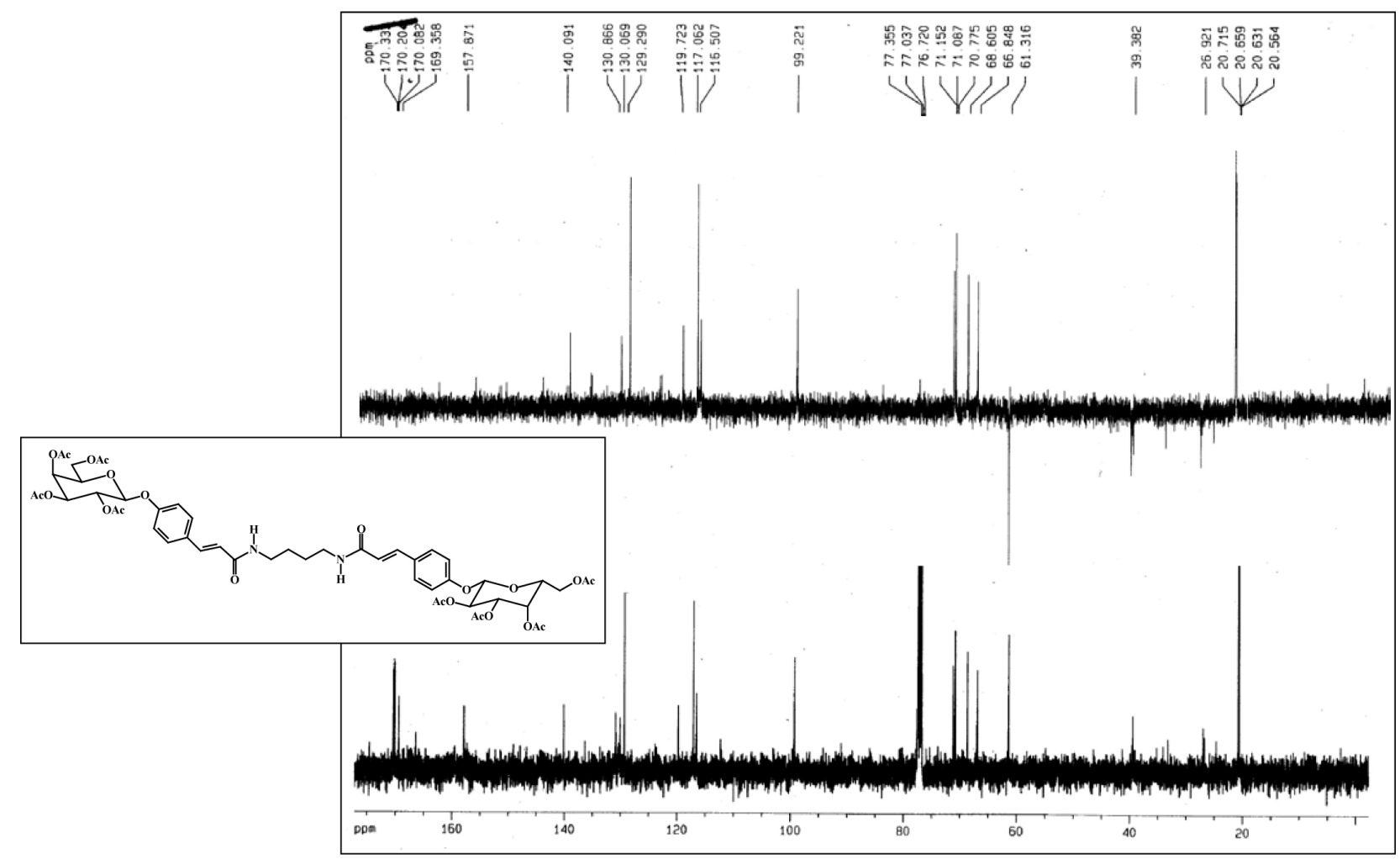

Figura 9S. Espectro de $\mathrm{RMN} \mathrm{de}{ }^{l} \mathrm{H}$ de $5\left(\mathrm{CDCl}_{3}, 400 \mathrm{MHz}\right)$

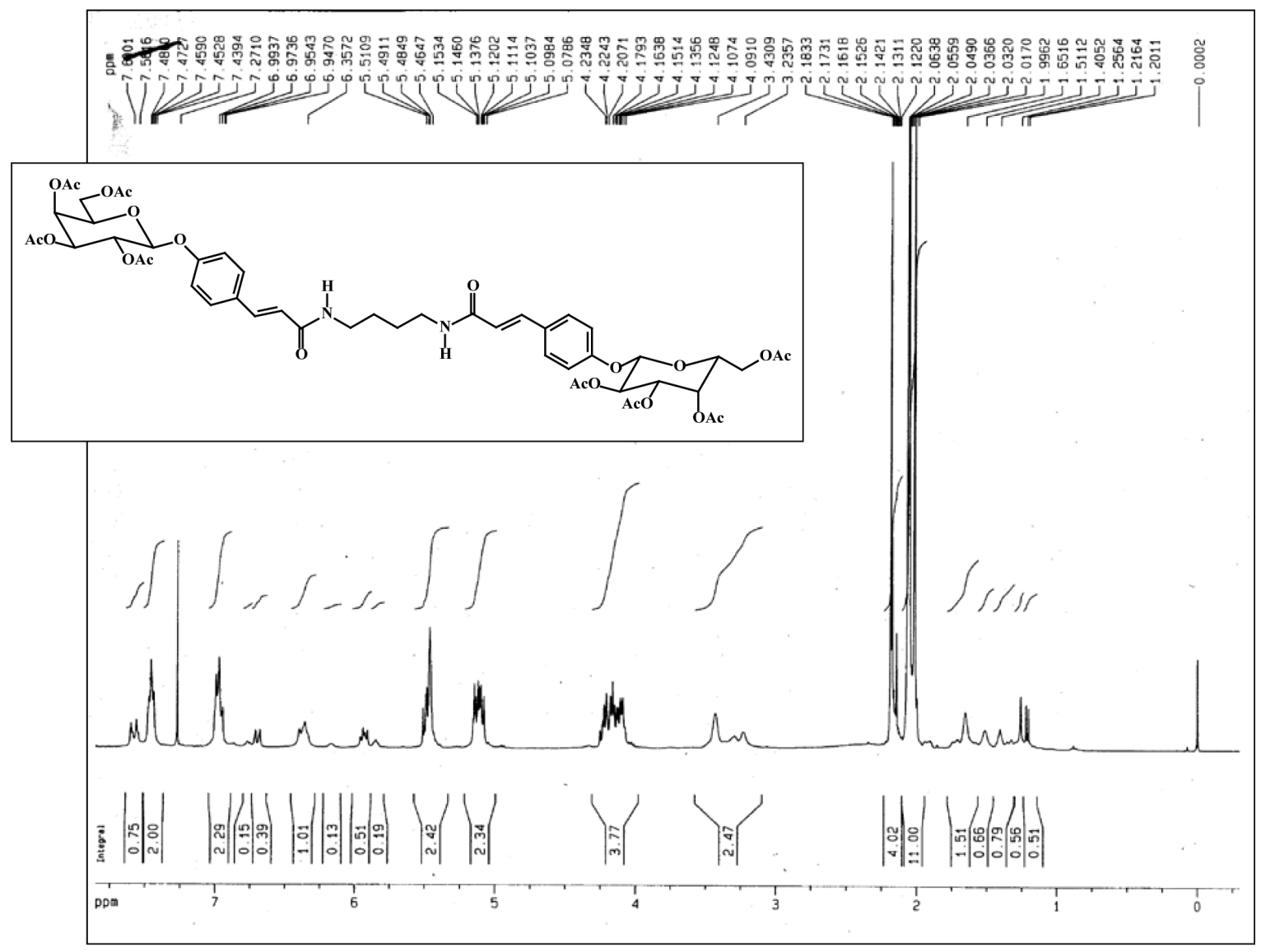

Figura 10S. Espectro de $\mathrm{RMN}$ de ${ }^{13} \mathrm{C}$ de $5\left(\mathrm{CDCl}_{3}, 100 \mathrm{MHz}\right)$ 


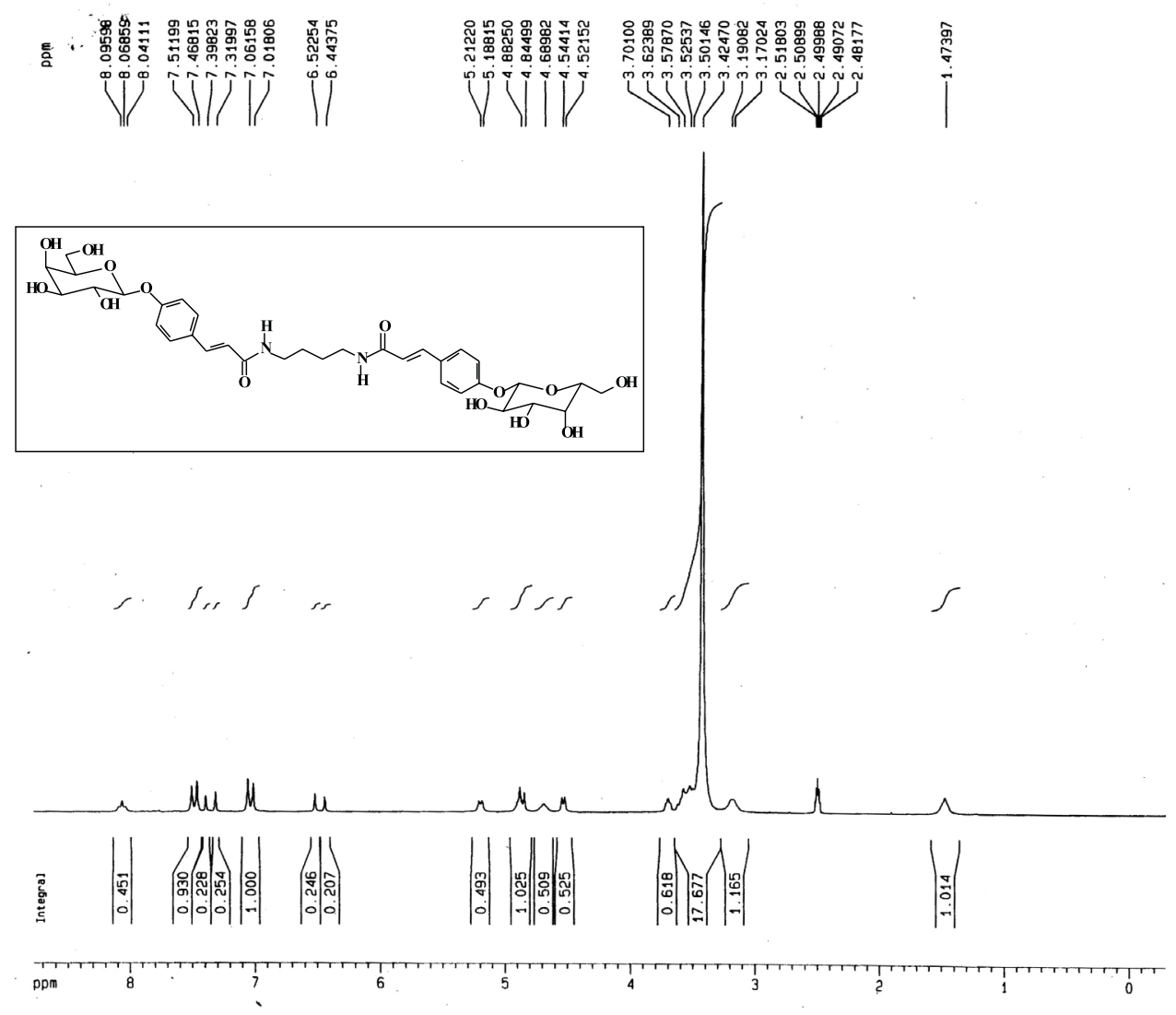

Figura 11S. Espectro de RMN de ${ }^{l} \mathrm{H}$ de $6\left(\mathrm{DMSO}_{6} \mathrm{~d}_{6}, 400 \mathrm{MHz}\right)$

|

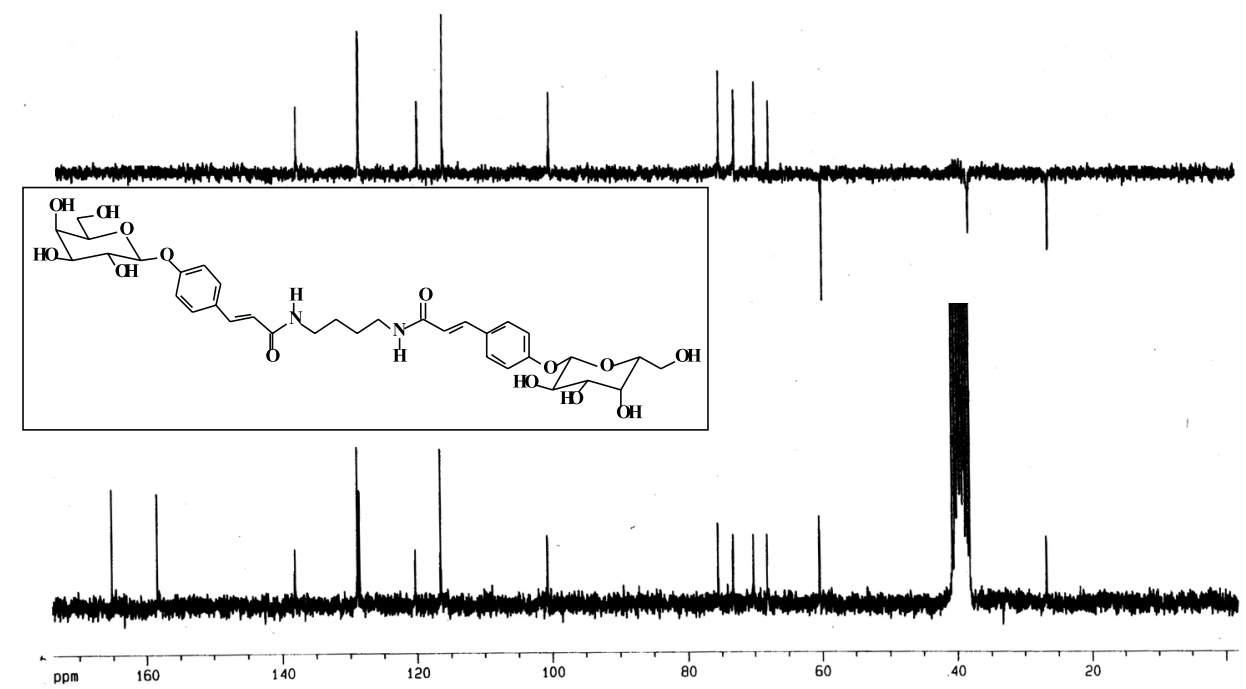

Figura 12S. Espectro de $\mathrm{RMN}$ de ${ }^{13} \mathrm{C}$ de $6\left(\mathrm{DMSO}_{-} \mathrm{d}_{6}, 100 \mathrm{MHz}\right)$ 


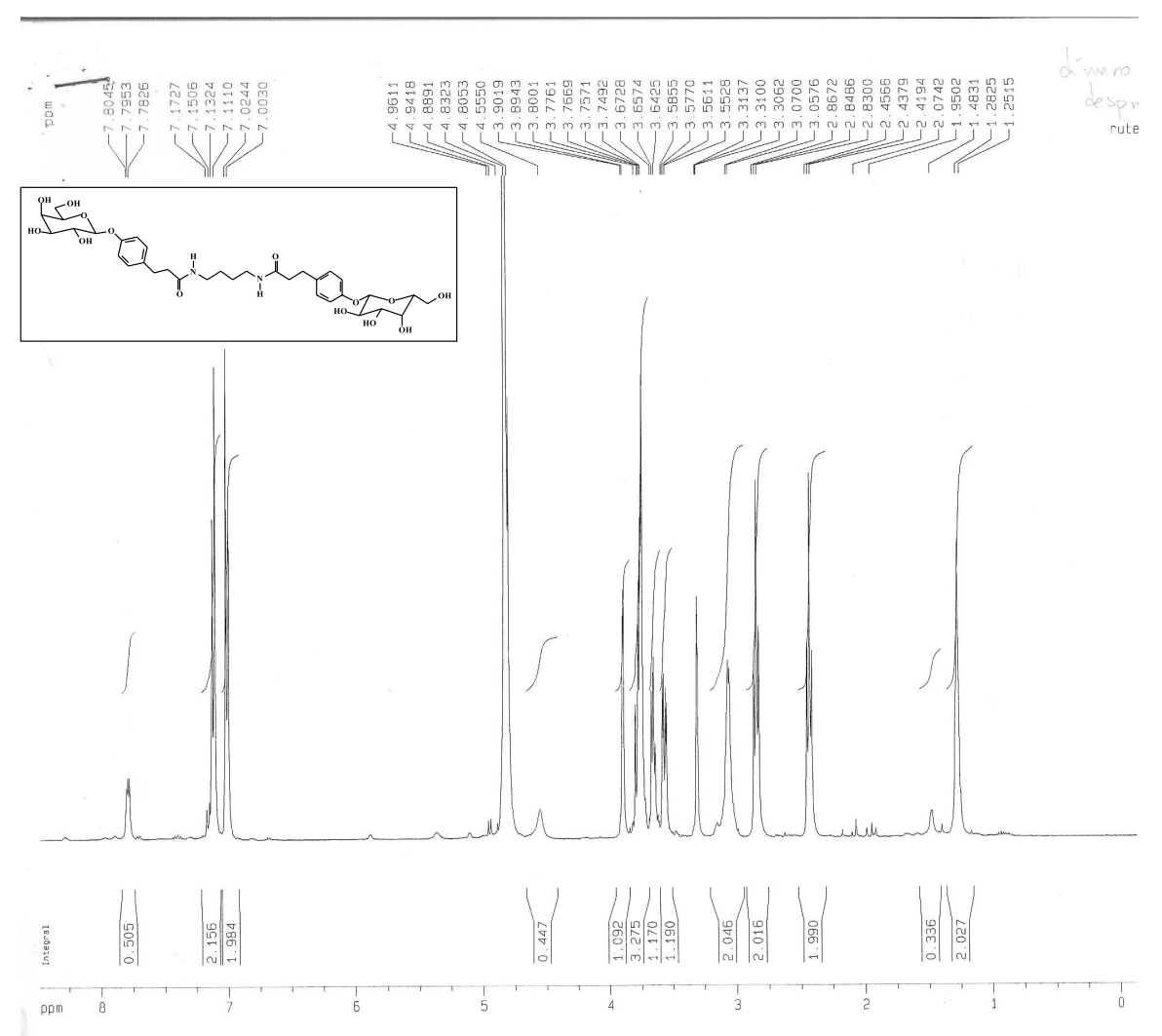

Figura 13S. Espectro de $\mathrm{RMN}$ de ${ }^{13} \mathrm{C}$ de $7(\mathrm{MeOD}, 400 \mathrm{MHz})$

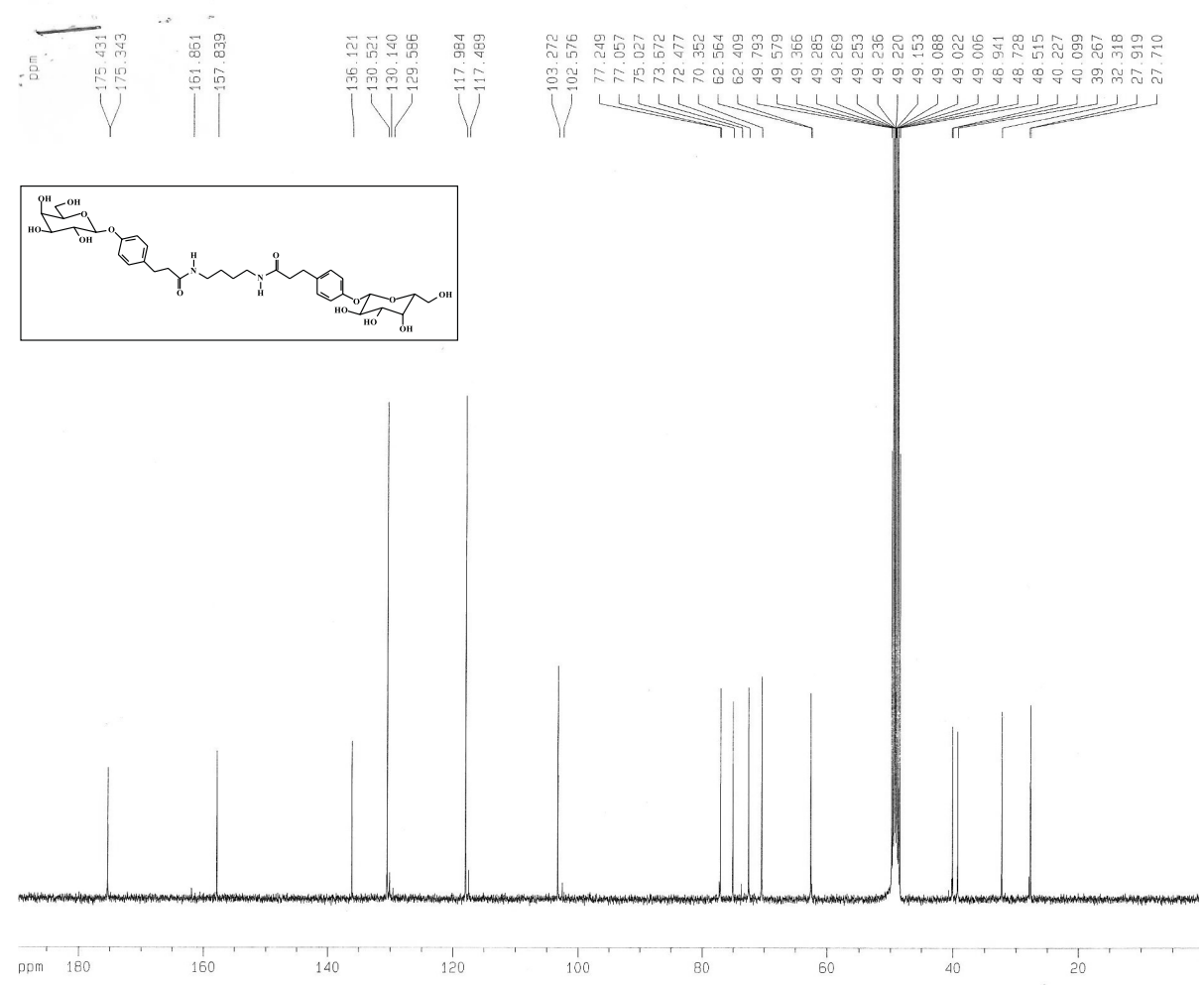

